\title{
Distributed Algorithms for End-to-End Packet Scheduling in Wireless Ad Hoc Networks
}

\author{
V. S. ANIL KUMAR and MADHAV V. MARATHE, Virginia Tech \\ SRINIVASAN PARTHASARATHY, IBM T. J. Watson Research Center \\ ARAVIND SRINIVASAN, University of Maryland, College Park
}

Packet scheduling is a particular challenge in wireless networks due to interference from nearby transmissions. A distance-2 interference model serves as a useful abstraction here, and we study packet routing and scheduling under this model of interference. The main focus of our work is the development of fully distributed (decentralized) protocols. We present polylogarithmic/constant factor approximation algorithms for various families of disk graphs (which capture the geometric nature of wireless-signal propagation), as well as near-optimal approximation algorithms for general graphs. A basic distributed coloring procedure, originally due to Luby [1993] (Journal of Computer and System Sciences, 47:250-286, 1993), underlies many of our algorithms. The work of Finocchi et al. [2002] (Proc. ACM-SIAM Symposium on Discrete Algorithms, 2002) showed that a natural modification of this algorithm leads to improved performance. A rigorous explanation of this was left as an open question, and we prove that the modified algorithm is indeed provably better in the worst case.

\section{CCS Concepts: • Theory of computation $\rightarrow$ Scheduling algorithms}

Additional Key Words and Phrases: Packet scheduling, wireless interference, distributed algorithms

ACM Reference Format:

V. S. Anil Kumar, Madhav V. Marathe, Srinivasan Parthasarathy, and Aravind Srinivasan. 2016. Distributed algorithms for end-to-end packet scheduling in wireless ad hoc networks. ACM Trans. Algorithms 12, 3, Article 28 (April 2016), 25 pages.

DOI: http://dx.doi.org/10.1145/2812811

\section{INTRODUCTION}

Packet routing and scheduling are two key problems that arise in the control and design of packet-switched networks. To send a packet from a node $u$ to node $v$ in a

V. S. Anil Kumar and Madhav Marathe have been supported in part by DTRA Grant HDTRA1-11-1-0016, DTRA CNIMS Contract HDTRA1-11-D-0016-0010, NSF Career CNS 0845700, NSF ICES CCF-1216000, NSF NETSE Grant CNS-1011769, and DOE DE-SC0003957. Srinivasan Parthasarathy has been supported in part by NSF Awards CCR-0208005 and ITR CNS-0426683. Aravind Srinivasan has been supported in part by NSF Awards CCR-0208005, ITR CNS-0426683, CNS-0626636, CNS-1010789, and CCF 1422569.

A preliminary version of this work appeared as "End-to-End Packet Scheduling in Wireless Ad-Hoc Networks" in the Proceedings of the ACM-SIAM Symposium on Discrete Algorithms, pages 1014-1023, 2004.

Authors' addresses: V. S. Anil Kumar and M. V. Marathe, Biocomplexity Institute of Virginia Tech, 1015 Life Science Circle, Blacksburg, VA 24061; emails: \{akumar, mmarathe\}@vbi.vt.edu (Most of the work was done when the authors were at the Los Alamos National Laboratory); S. Parthasarathy, IBM T. J. Watson Research Center, Yorktown Heights, NY; email: spartha@us.ibm.com (Most of this work was done while visiting the Los Alamos National Laboratory, and also while at the University of Maryland, College Park); A. Srinivasan, Department of Computer Science and Institute for Advanced Computer Studies, University of Maryland, College Park, MD 20742; email: srin@cs.umd.edu.

Permission to make digital or hard copies of part or all of this work for personal or classroom use is granted without fee provided that copies are not made or distributed for profit or commercial advantage and that copies show this notice on the first page or initial screen of a display along with the full citation. Copyrights for components of this work owned by others than ACM must be honored. Abstracting with credit is permitted. To copy otherwise, to republish, to post on servers, to redistribute to lists, or to use any component of this work in other works requires prior specific permission and/or a fee. Permissions may be requested from Publications Dept., ACM, Inc., 2 Penn Plaza, Suite 701, New York, NY 10121-0701 USA, fax +1 (212) 869-0481, or permissions@acm.org.

(c) 2016 ACM 1549-6325/2016/04-ART28 $\$ 15.00$

DOI: http://dx.doi.org/10.1145/2812811 


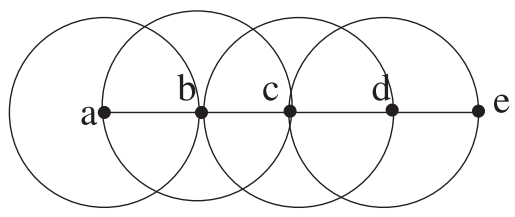

Fig. 1. The distance-2 interference: transmissions on $(a, b)$ and $(c, d)$ would cause interference, while transmissions on $(a, b)$ and $(d, e)$ are simultaneously possible in this model.

network, one needs to choose a path from $u$ to $v$; once the paths for all the packets have been determined, we are left with the issue of scheduling the packets along the paths. If multiple packets reach a node simultaneously, they must be queued or dropped. At most a given number of packets can be sent at a time on an edge (and sometimes nearby edges cannot have traffic simultaneously), and the scheduling problem is to decide which of the packets queued at a node should be sent first. The exact algorithms used for these problems have a profound impact on network performance. In this article, we study the scheduling problem in the context of wireless ad hoc networks. An ad hoc network consists of a group of transceivers (also known as stations) sharing a common wireless channel and communicating with each other using this channel. Our two foci here are dealing with interference and the development of distributed algorithms that have provably good approximation guarantees. Concretely, we are given a graph $G=(V, E)$, with packets that need to be sent from some sources to some destinations. We will also assume that paths are given; for the scheduling algorithms that we use, the algorithm of Srinivasan and Teo [2001] can be modified to obtain good paths.

The key issue in our case is interference, which implies that transmission by nearby links causes conflicts. An example of this is shown in Figure 1: if the transmissions on $(a, b)$ and $(c, d)$ occur simultaneously, node $b$ receives garbled signals from both $a$ and $d$ simultaneously; note that nodes $a$ and $c$ are not aware of this problem at $b$ (unlike, for instance, in Ethernet, where a collision is immediately detected by all nodes). This is called the hidden-node problem in wireless networks. On the other hand, the transmissions on $(a, b)$ and $(d, e)$ in Figure 1 can happen simultaneously. To model this notion combinatorially, we first construct the transmission graph for a set of transceivers by having a node for each radio, and an edge $(u, v)$ if $v$ lies in the transmission range of $u$ (note that we are assuming equal transmission ranges here; see Section 2 for the general versions that we work with). We now require that the set of edges on which simultaneous transmission happens forms a distance-2 matching (these definitions are given in Section 2$)$; edges $(a, b)$ and $(d, e)$ in Figure 1 satisfy this property. This model is called the distance-2 interference model, as in Balakrishnan et al. [2004] and Kumar et al. [2005]. Some earlier wireless network models, for example, by Ramanathan [1993, 1999], Ramanathan and Lloyd [1993], and Krumke et al. [2001], only placed vertex constraints - nodes that transmit simultaneously must form an independent set-which is clearly inadequate from the previous description. This requirement of distance-2 matchings might seem too restrictive sometimes: for instance, in Figure $1, b$ could transmit to $a$ and $c$ could transmit to $d$ simultaneously; this is called the exposed-node problem in radio networks. While this is true, reliable transmission requires transmission both ways (to acknowledge receipt of packets, for instance), which imposes the distance-2 scheduling constraint.

The discussion so far only addresses the restrictions that the Media Access Control (MAC) layer places on radio networks. The MAC layer only ensures delivery of packets from a node to its immediate neighbor. The Routing Layer of the wireless protocol 
stack is responsible for choosing the routes along which packets must travel, and the MAC layer moves packets one step at a time along these paths (see, e.g., Peterson and Davie [2000]). By incorporating the aforementioned interference constraint, we have the following generalization of the problem studied by Leighton et al. [1994], which we call End-to-End PACKet Scheduling with Interference (EPSI): given a wireless network with packets that have preassigned paths, develop a protocol to schedule the movement of the packets from their sources to their destinations, while ensuring that at each step, edges along which transmissions occur form a distance-2 matching. Our goal is to minimize the makespan, the maximum time taken by any packet to reach its destination. Also, these algorithms need to be distributed in order to be useful in practice. We remark that the problem of routing and scheduling using "standard" (i.e., distance-1 as opposed to our distance-2) matchings has been studied by Alon et al. [1994].

Our main contributions are described next.

(a) Packet-Scheduling Algorithms. We present the first provably efficient distributed algorithms for packet scheduling to minimize the makespan in networks with the distance-2 interference constraint. While the original motivation for the model is radio networks, we also study this problem on different nongeometric graph classes. Let $n$ denote the number of nodes in the given network. For arbitrary graphs with maximum degree $\Delta$, we present a distributed $O\left(\Delta \log ^{2} n / \log \log n\right)$-approximation algorithm; we also show that it is hard to approximate the minimum time within a factor of $\Delta^{1-\epsilon}$ for any constant $\epsilon>0$, even in the centralized, polynomial-time setting. Disk graphs, defined in Section 2, model radio communication well, and we obtain the following improved approximation algorithms for them. For general disk graphs, we present a distributed $O\left(\left(1+\log \frac{r_{\max }}{r_{\min }}\right) \cdot \log ^{2} n / \log \log n\right)$-approximation algorithm where $r_{\max }$ and $r_{\min }$ are the maximum and minimum node transmission radii, respectively, and a centralized $O(\log n / \log \log n)$-approximation algorithm. For unit-disk graphs, we present a distributed $O\left(\log ^{2} n / \log \log n\right)$-approximation algorithm and a centralized $O(1)$-approximation algorithm. The previous distributed algorithms are in the synchronous model; for unit-disk graphs, we also obtain a distributed $O\left(\log ^{2} n\right)$ approximation in an asynchronous model. Our results for unit-disk graphs can also be extended to a more general class of graphs known as $(r, s)$-civilized graphs. ${ }^{1}$ As discussed by Krumke et al. [2001] and Balakrishnan et al. [2004], $(r, s)$-civilized graphs are a more realistic model for ad hoc networks due to their ability to model occlusions.

A key driver of our algorithms is the random delays technique of Leighton et al. [1994]; we combine this with additional new ideas to develop our algorithms. In particular, the standard lower bound of "congestion plus dilation" of Leighton et al. [1994] which is introduced in the "Related Work" discussion toward the end of this section-is shown to be no longer suitable in our model, and we use a more refined lower bound. For general disk graphs, we introduce a new packing result, which, along with the graph-theoretic notion of inductive coloring, helps us claim our approximation bounds. Many of our algorithms also employ a basic (list-)coloring algorithm due to Luby [1993]; we prove the improved performance of a variant of this algorithm, as discussed next.

(b) Distributed Coloring and Network Decomposition. Variants of the aforementioned algorithm due to Luby [1993] have been useful in many distributed coloring

\footnotetext{
${ }^{1}$ For each fixed pair of real values $r>0$ and $s>0$, a graph $G$ can be drawn in $R^{d}$ in an $(r, s)$-civilized manner if its vertices can be mapped to points in $R^{d}$ so that the length of each edge is at most $r$, the distance between any two points is at least $s$, and no two edges intersect (except at their endpoints), as discussed in Krumke et al. [2001].
} 
algorithms (see, e.g., Grable and Panconesi [2000]), as well as in our algorithms for packet scheduling. Due to its simplicity and generality, an extensive experimental evaluation of this and related coloring algorithms has been undertaken by Finocchi et al. [2002]. A natural "nonlazy" variant of the original algorithm of Luby [1993], that is, where nodes do not sleep in any round, was empirically found to be much better. To quote Finocchi et al. [2002], "In particular, when compared with Luby's algorithm, it consistently turned out to be 2-3 times faster. The available asymptotic analyses do not explain this behavior ... and we leave it as an interesting open question." We present such a rigorous explanation regarding the worst-case behavior of the two algorithmswe exhibit a constant $c_{0}$ such that with probability $1-o(1)$ : (1) for any $n$-vertex graph, the modified algorithm terminates within $c_{0} \log n$ rounds, and (2) the original algorithm of Luby [1993] requires $\left(c_{0}+\Omega(1)\right) \log n$ rounds when run on the complete graph $K_{n}$. We note that this analysis holds for vertex-based interference (see, e.g., Ramanathan [1993, 1999] and Ramanathan and Lloyd [1993]), and not the distance-2 interference model considered in our earlier results, which is more challenging. In passing, we also improve the running time of a powerful distributed primitive known as network decomposition (see, e.g., the work by Awerbuch et al. [1994, 1989], Awerbuch and Peleg [1992], Linial and Saks [1993], and Panconesi and Srinivasan [1996]): we observe that the protocol of Linial and Saks [1993], which runs in $O\left(\log ^{2} n\right)$ time, can be modified to run in $O(\log n)$ time. (See, e.g., Dubhashi et al. [2005] for an application of network decomposition to distributed routing protocols for wireless ad hoc networks.)

Related Work. There are broadly two classes of models for wireless interference, one using a graph-based notion, as we study here, and another based on the signal-tointerference and noise ratio (SINR); see Lotker and Peleg [2010], Halldórsson and Tonoyan [2015], and Kumar et al. [2008] for discussion of these two models. The SINR model is much more challenging to analyze because of its nonlocal nature, and graphbased models remain popular in the wireless networking literature. Halldórsson and Tonoyan [2015] study the relationship between these two types of models, specifically on how the feasibility in one model is related to feasibility in the other. Link scheduling in wireless networks is a very active area, and there has been a lot of work in both centralized and distributed algorithms in all types of interference models (see, e.g., Dinitz [2010], Halldórsson and Mitra [2011], Dams et al. [2012], Pei and Vullikanti [2012], Kesselheim [2012], and Balakrishnan et al. [2004]).

The end-to-end scheduling problem is much more challenging, and for the classical model of "one packet per edge at a time," one of the most significant results is the work of Leighton et al. [1994], which shows using the Lovász Local Lemma that a simple lower bound on the makespan is correct up to some universal constant. Their result assumes that the packets already come with prespecified paths. More precisely, suppose $C$ is the congestion (the maximum number of routing paths that pass through any particular edge in the graph), and $D$ is the dilation (maximum length of any routing path). It is easy to see that any schedule needs at least $\max \{C, D\}$ time-steps in this "one packet per edge at a time" wireline model. The very surprising result of Leighton et al. [1994] is that $O(C+D)$ steps always suffice. This work was followed by a series of papers improving either the performance or the computational model (see, e.g., Leighton et al. [1999], Scheideler [1998], auf der Heide and Vöcking [1995], Andrews et al. [1997], Andrews [2000], and Ostrovsky and Rabani [1997]), including distributed scheduling for various kinds of network models and objectives for wireline networks and wireless networks with restricted interference models. Tradeoffs between congestion, dilation, and energy for routing and scheduling in wireless networks have also been studied (e.g. Ruhrup et al. [2003] and auf der Heide et al. [2004]). One of the main challenges in wireless network is the interference model. Our results show that the definition of 
$C$ will have to be modified significantly in order to get a good lower bound in wireless networks with general interference models.

Other objectives that capture latency have also been studied, for example, the flow time, which is defined in the following manner. Each packet $j$ is associated with a release time, denoted by $r_{j}$, which is the time when it becomes available. The flow time is defined as $\max _{j}\left(C_{j}-r_{j}\right)$, where $C_{j}$ denotes the completion time of packet $j$. Bonifaci et al. [2011] develop constant factor approximation algorithms for minimizing the flow time for aggregation (i.e., at a common sink) in wireless networks. Our results give the first distributed polylogarithmic approximation algorithms for end-to-end packet scheduling in wireless networks. Following the conference version of this article [Kumar et al. 2004], our approach has been extended to a number of other interference models for wireless networks, including the SINR model, such as Fanghänel et al. [2009], and Chafekar et al. [2007]. These papers have developed a notion of congestion that allows the problem to be reduced to our approach. Chafekar et al. [2007] define a congestion measure that considers SLTs of comparable length, leading to an approximation bound that depends on the aspect ratio, that is, the ratio of the lengths of the longest to the shortest link. Fanghänel et al. [2009] define a slightly different notion of congestion, which leads to better approximation bounds, which hold for other metrics than just Euclidean. A unique aspect of the SINR model is power control, which requires specifying the transmission power level for each link. Inductive ordering has been a successful approach for the link scheduling problem (without any end-to-end constraints) in many variants of this problem in the SINR model-see, for example, Halldórsson and Wattenhofer [2009] and Wan et al. [2009].

\section{PRELIMINARIES}

We now present some basic definitions about transmission graphs and graph-theoretic distance, and then discuss our basic scheduling problem.

We will work with transmission graphs $G(V, E)$, which specify the edges along which transmission can take place; these graphs will be undirected for most graph classes considered here, with the exception of disk graphs. A disk graph is specified by a planar set of points $V$, with a disk $\mathcal{D}(v)$ of radius $r(v)$ centered at each $v \in V$. The directed graph $G(V, E)$ induced by these disks is the following: the set of nodes is $V$ and a (directed) edge $(u, v)$ is present if $v \in \mathcal{D}(u)$. The special case where all radii are equal is called a unit-disk graph, and in this case, $(u, v) \in E$ if and only if $(v, u) \in E$; thus, we can view unit-disk graphs as undirected. Section 4 discusses the properties of disk graphs in more detail.

If $G(V, E)$ is undirected, we will usually let $\Delta=\Delta(G)$ denote the maximum degree of $G$. Given subsets $A, B \subseteq V$, the distance $d_{G}(A, B)$ is defined to be the minimum length of the (directed) shortest path over all pairs of vertices $(a, b) \in A \times B$. We emphasize that the notions of "distance" and "length" here are the usual graph-theoretic ones, where we count one for every edge in a path; in particular, these graph-theoretic distances $d_{G}(\cdot, \cdot)$ are distinct from the geometric notion of radii $r(v)$. For edges $e=(u, v)$ and $e^{\prime}=\left(u^{\prime}, v^{\prime}\right)$ of $G, d_{G}\left(e, e^{\prime}\right)$ is defined as $d_{G}\left(\{u, v\},\left\{u^{\prime}, v^{\prime}\right\}\right)$. For a vertex $w \in V$, we will sometimes use $d_{G}(w, e)$ to denote $d_{G}(\{w\},\{u, v\})$; we drop the subscript $G$ whenever it is clear from the context. A subset $M \subset E$ is said to be a distance-2 matching if $d\left(e, e^{\prime}\right) \geq 2$ for any distinct pair $e, e^{\prime} \in M$.

Formally, an instance of our packet-scheduling problem is specified as $\operatorname{EPSI}\left(G(V, E),\left\{p_{1}, p_{2}, \ldots, p_{k}\right\}\right)$, where (1) $G(V, E)$ is the underlying transmission graph, and (2) the packets to be transmitted are $p_{1}, p_{2}, \ldots, p_{k}$, with packet $p_{i}$ starting at $s_{i}$ and destined for $t_{i}$, to be routed along the path $P_{i}$; the path $P_{i}$ is encoded in packet $p_{i}$. We will assume without loss of generality that each $P_{i}$ is simple. Because of our communication model, which requires two-way transmission as discussed in Section 1, only bidirected 
edges can be used for transmission even if the graph is directed, so we assume that the given paths $P_{1}, P_{2}, \ldots, P_{k}$ only use bidirected edges. The unidirected edges (those with no "reverse" edges) only contribute to the interference: therefore, if edge $e=(u, v)$ is being used at time $t$, no other edge $e^{\prime}=\left(u^{\prime}, v^{\prime}\right)$ with $\min \left\{d\left(u^{\prime}, e\right), d\left(v^{\prime}, e\right), d\left(u, e^{\prime}\right), d\left(v, e^{\prime}\right)\right\} \leq 1$ can be used simultaneously. (We may assume that each time slot is a virtual slot composed of two time slots: the first for transmissions, and the second to acknowledge the successful ones.) We will assume that any packet takes one unit of time to cross a link and at any time at most one packet can cross a link. In addition, if packets are being sent simultaneously on edges $e=(u, v)$ and $e^{\prime}=\left(u^{\prime}, v^{\prime}\right)$, then $d\left(e, e^{\prime}\right) \geq 2$. If a packet transmission violates either of these requirements (i.e., if some other transmission is made simultaneously on an edge that is within distance less than two), then the transmission fails and has to be retried later. Each node/edge has a buffer in which a packet can wait until it successfully moves to the next node in its path. The objective is to construct a schedule $\mathcal{S}$ that decides which packet should be sent out at a node at any time. A schedule is valid if and only if it sends all packets along their paths subject to the aforementioned retrial requirement (in the case of failures). Let $C_{\mathcal{S}}\left(p_{i}\right)$ denote the time at which packet $p_{i}$ is delivered in schedule $\mathcal{S}$; this can be a random variable if our scheduling protocol is randomized. The makespan of $\mathcal{S}$, denoted by $C(\mathcal{S})=\max _{i} C_{\mathcal{S}}\left(p_{i}\right)$, is the time taken by $\mathcal{S}$ to route all the packets, and our objective is to construct a schedule with low makespan. For the most part, we will assume a synchronous, distributed communication model; this is reasonable in our context of a primarily local area network, since nodes can keep synchronized, for example, by using GPS receivers.

Notation. The abbreviation "w.h.p." for "with high probability" will be used to denote probabilities that are at least $1-n^{-a}$, where $a$ can be made any desired constant for sufficiently large $n$ by increasing the other constant parameters in our algorithm. (As usual, $n$ here denotes the number of nodes in the network.)

\section{PACKET SCHEDULING USING RANDOM INITIAL DELAYS}

Motivated by the "random initial delays" idea of Leighton et al. [1994], we now set up a framework for packet scheduling that will be useful in Sections 4, 5, and 6.

\subsection{Single-Link Transmissions}

Let us first describe three basic scheduling strategies for single-link transmissions. Suppose we have a collection $\mathcal{A}$ of single-link transmissions to be scheduled. Suppose further that the interference model is given by an undirected graph $H=(\mathcal{A}, E)$ : a transmission $a \in \mathcal{A}$ scheduled at time slot $t$ is successful in that time slot if and only if no neighbor $a^{\prime}$ of $a$ in $H$ is also scheduled at time $t$. Let $\sigma$ be some ordering of $\mathcal{A}$ such that each $a \in \mathcal{A}$ has at most $\alpha$ neighbors preceding it in $\sigma$; let $\beta$ denote the maximum degree of $H$. Then, the three scheduling approaches are as follows.

Greedy. This is the classical (centralized) strategy that uses $\Delta(H)+1$ colors for coloring a graph, which corresponds to $\beta+1$ time slots. Process the $a \in \mathcal{A}$ in any sequential order; when handing $a$, assign to $a$ any time slot among the ones given that has not yet been assigned to any of its neighbors.

Inductive $(\sigma)$. This centralized strategy uses $\alpha+1$ time slots. Process the $a \in \mathcal{A}$ in the order $\sigma$; when handling $a$, assign to $a$ any time slot among the ones given that has not been assigned to any of its neighbors that precedes a in $\sigma$. 
Distributed $(N)$. Given a parameter $N \geq|\mathcal{A}|$, this distributed approach schedules all the transmissions successfully within $\ell=\lceil e(\beta+1) \ln N\rceil$ time slots, ${ }^{2}$ with probability at least $1-|\mathcal{A}| / N$. The idea is, for $t=1,2, \ldots, \ell$, to schedule each yet-unsuccessful $a \in \mathcal{A}$ independently with probability $1 /(\beta+1)$ at time slot $t$. As for the analysis, let us fix $a \in \mathcal{A}$ and show now that the probability of $a$ never succeeding is at most $1 / N$; a union bound over all the $a$ will then complete the proof. Note that regardless of past history, the probability of a yet-unsuccessful $a$ succeeding at a time slot $t$ is at least

$$
p \doteq \frac{1}{\beta+1} \cdot\left(1-\frac{1}{\beta+1}\right)^{\beta} \geq \frac{1}{\beta+1} \cdot \frac{1}{e} .
$$

Thus, the probability of $a$ never succeeding is at most $(1-p)^{\ell} \leq e^{-p \ell} \leq 1 / N$, as required.

\subsection{Scheduling Along Routing Paths}

Now suppose we are in the general case where we have $k$ routing paths $P_{1}, P_{2}, \ldots, P_{k}$ in some $n$-vertex graph $G$, each of length at most $D$; we will view each $P_{i}$ as being a sequence $\left\langle t_{i, 0}, t_{i, 1}, \ldots,\right\rangle$ of at most $D$ single-link transmissions (SLTs). Suppose the list of all SLTs has been partitioned into $A_{1}, A_{2}, \ldots, A_{q}$ and a function $I_{i}: A_{i} \rightarrow 2^{A_{i}}$ has been defined for each $i$, with a parameter $C^{*}$ such that $\max _{i, a \in A_{i}}\left|I_{i}(a)\right| \leq C^{*}$. As we discuss later, $I_{i}(a)$ will be taken to be the set of SLTs $a^{\prime} \in A_{i}-\{a\}$ that interfere with $a$. A unifying approach to scheduling all of these transmissions via random initial delays is described later. We will also study a variant at the end of this subsection.

Depending on the model, we will choose an integer $L=L\left(C^{*}, n\right)$, whose value we will uncover later in the proof of Corollary 3.3, depending on whether the algorithm is centralized or distributed. Let the path $P_{i}$ be $\left\langle v_{i, 0}, v_{i, 1}, \ldots\right\rangle$; thus, the SLT $t_{i, j}$ is from $v_{i, j}$ to $v_{i, j+1}$. The initialization and key invariant (which hold with high probability) of our scheduling algorithm are as follows:

Initialization: Give each packet $p_{i}$ (which needs to use path $P_{i}$ ) an independent random value $Y_{i}$ in the range $\left\{0,1, \ldots, C^{*}-1\right\} ; p_{i}$ waits at its source $s_{i}$ until time slot $Y_{i} \cdot L$.

Invariant: Each packet $p_{i}$ reaches the $j$ th node $v_{i, j}$ on $P_{i}$ by time $\left(Y_{i}+j\right) \cdot L$. If it reaches $v_{i, j}$ before this time slot, it remains inactive and becomes active only after this time slot.

Assuming that the time slots begin at 0 , a frame is a sequence of $L$ consecutive time slots that begins at a slot that is a multiple of $L$. Note that our scheduling happens frame by frame, where each frame involves a collection of SLTs that come from different packets. In particular, the $j^{\text {th }}$ transmission of packet $p_{g}$ will get scheduled in frame $F=\langle T \cdot L, T \cdot L+1, \ldots,(T+1) \cdot L-1\rangle$ if and only if $Y_{g}+j=T$, that is, if and only if

$$
Y_{g}=T-j \text {. }
$$

We need to present the frame-scheduling algorithm and prove by induction on $j$ that the invariant holds (with high probability for an appropriately large $L$ ); the base case $j=0$ is immediate. Setting $j=D$ will then show that since $Y_{i}<C^{*}$, the total makespan is at most $\left(C^{*}+D\right) \cdot L$. The analysis and the resultant choice of an appropriate $L$ proceed

\footnotetext{
${ }^{2}$ The dependence on $\beta$ in the number of time slots is eliminated by Luby's algorithm [Luby 1993] discussed in Section 7. However, that algorithm works in a different model in which nodes can exchange information (about "tentatively chosen colors") with all of their neighbors in a single time slot. In contrast, the model here requires no communication between the different $a \in \mathcal{A}$; all we require is that a packet can recognize when it has been transmitted successfully.
} 
as follows. Also, note that because of our invariants, each packet is queued up on an edge for at most $O(L)$ time, so that the maximum queue size for any edge is $O\left(C^{*} L\right)$.

Recall the standard Chernoff-Hoeffding upper-tail bound:

FACt 3.1 (Chernoff [1952], HoefFding [1963]). Given independent random variables $X_{1}, X_{2}, \ldots, X_{t} \in[0,1]$, let $X=\sum_{i=1}^{t} X_{i}$ and $\mu=\mathbf{E}[X]$. Then, for any $\delta>0, \operatorname{Pr}[X \geq$ $\mu(1+\delta)] \leq G(\mu, \delta)$, where $G(\mu, \delta)=\left(e^{\delta} /(1+\delta)^{1+\delta}\right)^{\mu}$.

We next show:

LEMMA 3.2. Let $\lambda$ be a positive integer. The probability that there exists some frame $F$, some $A_{i}$, and some $S L T a \in A_{i}$ such that at least $\lambda$ elements of $I_{i}(a)$ get scheduled in $F$ is at most $k D\left(C^{*}+D\right) \cdot(e / \lambda)^{\lambda}$.

Proof. Fix a frame $F$, an $A_{i}$, and an SLT $a \in A_{i}$. The number of choices for $\left(A_{i}, a\right)$ is at most $k D$, and the number of choices for $F$ is at most $C^{*}+D$. Thus, it suffices to show that the "bad event" specified in the statement of the lemma happens for $\left(F, A_{i}, a\right)$ with probability at most $(e / \lambda)^{\lambda}$.

Index the elements $a^{\prime}$ of $I_{i}(a)$ by pairs of the form $(g, h)$, where the first index $g$ is the index of the packet $p_{g}$ that $a^{\prime}$ corresponds to, and the second index $h$ is such that the transmission $a^{\prime}$ corresponds to the SLT $t_{a^{\prime}, h}$. Let $X_{g, h}$ be the indicator random variable for the transmission $(g, h)$ getting scheduled in the frame $F$, and let $X_{g}=\sum_{h} X_{g, h}$. From the definition, observe that for any given $g$, there can be at most one value of $h$ such that $X_{g, h}=1$, so that $X_{g} \leq 1$. Then, $Z=\sum_{g} X_{g}$ denotes the number of elements of $I_{i}(a)$ that get scheduled in $F$. Because of the independent random delays, the $X_{g} \mathrm{~s}$ are independent.

Now, if $F=\langle T \cdot L, T \cdot L+1, \ldots,(T+1) \cdot L-1\rangle$, Equation (1) shows that $\mathbf{E}\left[X_{g, h}\right] \leq 1 / C^{*}$, since there is at most one choice of the random delay $Y_{g}$ that can cause $X_{g, h}$ to be 1 . Since $X_{g} \leq 1$ and $\left|I_{i}(a)\right| \leq C^{*}$, we get that $\mathbf{E}[Z] \leq 1$. Thus, by Fact 3.1 , we have that

$$
\operatorname{Pr}[Z \geq \lambda] \leq e^{\lambda-1} / \lambda^{\lambda} \leq(e / \lambda)^{\lambda}
$$

as required.

CoROLlary 3.3. The packet-scheduling problem has a centralized algorithm and a distributed algorithm that have the respective makespans of $O\left(\left(C^{*}+D\right) \cdot q \cdot \log n / \log \log n\right)$ and $O\left(\left(C^{*}+D\right) \cdot q \cdot \log ^{2} n / \log \log n\right)$, w.h.p.

Proof. We use Lemma 3.2 with each $I_{i}(a)$ defined as the set of SLTs $a^{\prime} \in\left(A_{i}-\{a\}\right)$ that interfere with $a$.

First, since $k D \cdot\left(C^{*}+D\right)$ is bounded by some fixed polynomial of $n$, we have, for any desired constant $c>0$, a constant $c^{\prime}$ such that $\lambda=c^{\prime} \log n / \log \log n$ will lead to the failure-probability bound in the statement of Lemma 3.2 being at most $n^{-c}$. Choose such a $\lambda$. A set $\mathcal{T}$ of transmissions in a frame $F$ is scheduled in the following manner, using the algorithms of Section 3.1.

-For $i=1$ to $q$ in turn, schedule the links in $\mathcal{T} \cap A_{i}$, so that all of these are completed within $L$ time slots.

- In the centralized approach, use algorithm Greedy in at most $\lambda+1$ time slots.

-In the distributed approach, use algorithm Distributed $\left(c^{\prime \prime} \log N\right)$ for some suitably large constant $c^{\prime \prime}$ (thus, the failure probability of this algorithm will be of the form $n^{-c}$ for any desired constant $\left.c\right)$ in $O(\lambda \log n)$ time slots.

The respective time bounds for the previous Greedy and Distributed algorithms follow from the discussion in Section 3.1, since $\lambda$ is the maximum number of interfering neighbors. Therefore, the scheduling of $\mathcal{T}$ is completed within $L=q(\lambda+1)$ and 
$L=O(q \lambda \log n)$ time slots in the Greedy and Distributed approaches, respectively. This completes the proof (via the obvious induction on $j$ in order to prove the invariant) since $\lambda=O(\log n / \log \log n)$, and since our total makespan is at most $\left(C^{*}+D\right) \cdot L$.

A Variant. Now consider a variant where we again have the routing paths $P_{1}, P_{2}, \ldots, P_{k}$ but where all the (at most $k D$ ) corresponding SLTs can be ordered according to some permutation $\sigma$, such that each such SLT $a$ has at most $C_{\text {ind }}^{*}$ other SLTs $a^{\prime}$ that precede $a$ in $\sigma$ and interfere with $a$. We show next that the results of Corollary 3.3 can be extended to this variant.

COROLlary 3.4. The packet-scheduling problem parameterized by $C_{\text {ind }}^{*}$ has a centralized algorithm that has a makespan of $O\left(\left(C_{\text {ind }}^{*}+D\right) \cdot \log n / \log \log n\right)$ w.h.p.

Proof (Sketch). The proof follows on the same lines as that of Corollary 3.3, and we sketch it here. The initialization and invariant are largely the same, except that in the former, we give each packet $p_{i}$ an independent random value $Y_{i}$ in the range $\left\{0,1, \ldots, C_{\text {ind }}^{*}-1\right\}$ : note that we use $C_{\text {ind }}^{*}$ instead of $C^{*}$ here.

Let $\mathcal{T}(F)$ denote the (random) set of SLTs that get assigned to the framescheduling problem corresponding to frame $F$ now. As in Corollary 3.3, a suitable $\lambda=O(\log n / \log \log n)$ guarantees, in exactly the same way as proven by Lemma 3.2, that w.h.p., for all $F$ and for all SLTs $a$, the number of $a^{\prime} \in \mathcal{T}(F)$ that precede $a$ in $\sigma$ is at most $\lambda$. Thus, letting $\sigma(F)$ denote the restriction of $\sigma$ to $\mathcal{T}(F)$, we can apply algorithm Inductive $(\sigma(F))$ of Section 3.1 and solve the problem for frame $F$ using $L=\lambda+1$ time slots, in a centralized manner. The bound for the makespan therefore follows, as in the proof of Corollary 3.3.

\section{EPSI ON DISK GRAPHS}

A popular model for radio networks is a disk graph. A disk around a point in space naturally corresponds to the effective transmission range of a radio located therein. As described in Section 2, we will view disk graphs as being directed. A packing result, Lemma 4.1, naturally leads to a generalized definition (Equation (3)) of congestion; we then employ the algorithms of Section 3.2. Our centralized scheduling algorithm yields an $O(\log n / \log \log n)$-approximation to the makespan, while our distributed scheduling algorithm leads to an $O\left(\left(1+\log \frac{r_{\max }}{r_{\min }}\right) \cdot \log ^{2} n / \log \log n\right)$-approximation, where $r_{\max }$ and $r_{\min }$ are the maximum and the minimum radii of the disks, respectively.

We need some further notation for disk graphs. Recall that since the graph is directed here, we may have $d(x, y) \neq d(y, x)$ for two entities (sets of nodes) $x$ and $y$ here. For edge $e=(u, v)$, define $r(e)=\max \{r(u), r(v)\}$. For a vertex $v$, define $N_{\text {ind }}(v)=$ $\left\{e^{\prime} \mid d\left(v, e^{\prime}\right)\right.$ or $\left.d\left(e^{\prime}, v\right) \leq 1, r\left(e^{\prime}\right) \geq r(v)\right\}$ and $N_{\text {ind }}(e)=\left\{e^{\prime} \mid d\left(e, e^{\prime}\right)\right.$ or $d\left(e^{\prime}, e\right) \leq 1, r\left(e^{\prime}\right) \geq$ $r(e)\}$. For any fixed $\alpha$, define $N_{\text {ind }}(\alpha, v)=\left\{e^{\prime} \mid d\left(v, e^{\prime}\right)\right.$ or $\left.d\left(e^{\prime}, v\right) \leq 1, r\left(e^{\prime}\right) \geq \alpha \cdot r(v)\right\}$ and $N_{\text {ind }}(\alpha, e)=\left\{e^{\prime} \mid d\left(e, e^{\prime}\right)\right.$ or $\left.d\left(e^{\prime}, e\right) \leq 1, r\left(e^{\prime}\right) \geq \alpha \cdot r(e)\right\}$. For the case of unit-disk graphs, we drop the subscript "ind" in all this notation.

In our given system of paths $\left\{P_{j}\right\}$, let $H(e, i)$ denote the number of times packet $i$ uses edge $e$ : since the $P_{j}$ are assumed to be simple, this number will be 0 or 1 . For any fixed value $\alpha$, define $C_{\mathrm{d}}(\alpha, e)$ to be the total number of times the edges in the set $N_{\text {ind }}(\alpha, e)$ are used by the packets: that is,

$$
C_{\mathrm{d}}(\alpha, e)=\sum_{e^{\prime} \in N_{\text {ind }}(\alpha, e)} \sum_{i} H\left(e^{\prime}, i\right) .
$$

Our key replacement for the standard notion of congestion now is

$$
C_{\mathrm{d}} \doteq \max _{e} C_{\mathrm{d}}(0.5, e) \text {. }
$$

$D$ is still defined to be the dilation, as before. 
Lemma 4.1. For any vertex $v$ and any constant $\alpha \in(0,1]$, the size of the largest distance- 2 matching in the subgraph induced by $N_{\text {ind }}(\alpha, v)$ is at most a constant whose value depends only on $\alpha$.

Proof. The proof is via a packing argument. Suppose $S=\left\{e_{1}, e_{2}, \ldots, e_{k}\right\}$ is a distance2 matching in $N_{\text {ind }}(\alpha, v)$. Let $S_{1} \subseteq S$ be the set of edges such that $d\left(v, e^{\prime}\right) \leq 1$ and let $S_{2}=S \backslash S_{1}$ (i.e., if $e \in S_{2}$, then $\left.\bar{d}\left(e^{\prime}, v\right) \leq 1\right)$. We will show that both $\left|S_{1}\right|$ and $\left|S_{2}\right|$ are $O(1)$; hence, we will have $|S|=O(1)$.

We first show that $\left|S_{1}\right|=O(1)$. Recall that $r(v)$ is the transmission range of $v$. Consider the disk $\mathcal{D}^{*}$ of radius $\left(1+\frac{3 \alpha}{2}\right) r(v)$ centered at vertex $v$. We will show that each edge $e \in S_{1}$ "occupies" a disjoint space of area of at least $\Omega\left((\alpha r(v))^{2}\right)$ in $\mathcal{D}^{*}$. Hence, $\left|S_{1}\right| \leq O\left(\left(\frac{1}{\alpha}+\frac{3}{2}\right)^{2}\right)=O(1)$ as claimed. Consider an edge $(p, q) \in S_{1}$. There are two possible cases:

Case 1: $r(p), r(q) \geq \alpha \cdot r(v)$. In this case, assume w.l.o.g. that $d(v, q) \leq 1$. Hence, $q$ is within a Euclidean distance of at most $r(v)$ from $v$.

Case 2: $r(p)<\alpha \cdot r(v)$ and $r(q) \geq \alpha \cdot r(v)$. Since $\min (d(v, p), d(v, q)) \leq 1$, node $q$ is at most a distance of $(1+\alpha) \cdot r(v)$ away from $v$.

(The third case where $r(q)<\alpha \cdot r(v)$ and $r(p) \geq \alpha \cdot r(v)$ is identical to the second and is ignored.)

In both the cases, consider a disk of radius $\frac{\alpha \cdot r(v)}{2}$ around $q$. We say that edge $(p, q)$ "occupies" this region of area $\frac{\pi(\alpha \cdot r(v))^{2}}{4}$. Crucially, no other edge $(f, g) \in S_{1}$ can "occupy" any of this region: otherwise, $q$ is within the range of either $f$ or $g$ and hence $\min \{d(q,(f, g)), d((f, g), q)\} \leq 1$, which violates the distance-2 constraint. Hence, the claim $\left|S_{1}\right|=O(1)$ follows.

We now show that $\left|S_{2}\right|=O(1)$. We first note that for any edge $(p, q) \in S_{2}, d((p, q), v) \leq$ 1. W.l.o.g., we assume that $d(q, v) \leq 1$. Consider an arbitrarily large disk centered at $v$ that contains all the vertices in the network. Consider a sector that subtends an angle of 60 degrees at $v$. Let $\|x-y\|$ denote the Euclidean distance between points $x$ and $y$. For any two points $x, y$ that lie in this sector, it is easy to see using simple geometry that $\|x-y\| \leq \max \{\|x-v\|,\|y-v\|\}$. Hence, if two nodes $q$ and $f$ exist in this sector such that edges $(q, v)$ and $(f, v)$ are both present, then there is an edge between these two nodes. In particular, this implies that at most one edge from $\left|S_{2}\right|$ can have an endpoint in the sector (without violating the distance-2 constraint). Since the disk can be partitioned into at most six disjoint sectors of angle 60 degrees each, $\left|S_{2}\right| \leq 6$.

LEMMA 4.2. $O P T=\Omega\left(C_{d}+D\right)$.

Proof. We just need to verify that $O P T=\Omega\left(C_{\mathrm{d}}\right)$. This follows from Lemma 4.1: at any time instant, the set of edges on which packets can be transmitted simultaneously forms a distance-2 matching. From Lemma 4.1, for any edge $e=(u, v)$, at most $O(1)$ edges can be simultaneously used within $N_{\text {ind }}(0.5, u)$ and within $N_{\text {ind }}(0.5, v)$. Therefore, $\Omega\left(C_{\mathrm{d}}(0.5, e)\right)$ timesteps are needed to transmit all the packets that use the edges in the set $N_{\text {ind }}(0.5, e)$.

Algorithms. Our distributed and centralized algorithms are as follows.

To conform with the notation used at the beginning of Section 3.2, we take:

$-q=1+\left\lfloor\log \frac{r_{\max }}{r_{\min }}\right\rfloor$

$-A_{j}$ as the set of SLTs that require edges $e$ whose $r(\cdot)$ values lie in the range $\left[2^{j-1}\right.$.

$\left.r_{\min }, 2^{j} \cdot r_{\min }\right) ;$ and

$-I_{i}(a)$ as the set of SLTs $a^{\prime} \in\left(A_{i}-\{a\}\right)$ that interfere with $a$. 
Our definition (Equation (3)) thus shows that we can take $C^{*}=C_{\mathrm{d}}$. The distributed algorithm is the one then given by Corollary 3.3. For the centralized algorithm, we appeal to the variant discussed toward the end of Section 3.2; the required order $\sigma$ on the SLTs is any order in which if $a$ precedes $a^{\prime}$ in $\sigma$, then the edges $e$ and $e^{\prime}$ respectively required by $a$ and $a^{\prime}$ satisfy $r(e) \geq r\left(e^{\prime}\right)$. Also note from Equations (2) and (3) that for all edges $e$,

$$
\sum_{e^{\prime} \in N_{\text {ind }}(1.0, e)} \sum_{i} H\left(e^{\prime}, i\right) \leq C_{\mathrm{d}} ;
$$

this is simply because $N_{\text {ind }}(1.0, e) \subseteq N_{\text {ind }}(0.5, e)$. Thus, in the notation of the variant discussed in Section 3.2, we can take $C_{\text {ind }}^{*}=C_{\mathrm{d}}$ for the permutation $\sigma$; the centralized algorithm is as given by Corollary 3.4. Summarizing:

THEOREM 4.3. The EPSI problem on disk graphs has centralized and distributed algorithms whose respective makespans are $O\left(\left(C_{d}+D\right) \cdot \log n / \log \log n\right)$ and $O\left(\left(C_{d}+D\right)\right.$. $\left.\left(1+\log \frac{r_{\max }}{r_{\min }}\right) \cdot \log ^{2} n / \log \log n\right), w \cdot h \cdot p$.

\section{EPSI ON UNIT-DISK GRAPHS}

When all disks have the same radius, we obtain significant improvements in the approximation guarantee. By a repeated planar decomposition, we can actually get an $O$ (1) approximation. This sort of decomposition only requires a sparsity condition, rather than geometry, and can be applied to bounded genus graphs also. We then give an $O(\log n)$ distributed algorithm by refining the analysis of the algorithm DiskEPS in Section 4. Finally, we give a distributed algorithm in the asynchronous model with a $O\left(\log ^{2} n\right)$ approximation guarantee.

\subsection{An $O(1)$-Approximation Algorithm}

The notation used here is defined in Section 2 . We assume the common radius to be 1 here. Let $B$ be a bounding box in the plane for the points in $V$. If we assume that $G$ is a connected graph, $B$ must have sides of length $O(n)$. Let $B_{\ell}^{0}$ be a partition of $B$ into smaller grid cells, each cell having dimensions $\ell \times \ell$. Let $B_{\ell}^{1}, B_{\ell}^{2}$, and $B_{\ell}^{3}$ be the partitions obtained by translating the grid $B_{\ell}^{0}$ by $\ell / 2$ along the x-axis, the y-axis, and both $\mathrm{x}$-and $y$-axes respectively. A cell in these partitions will refer to one of the $\ell \times \ell$-sized pieces in it, and a point in these partitions is any lattice point with integer coordinates. We will denote lattice points within by small letters and the $\ell \times \ell$ cells within by capital letters.

We use simplifications of the definitions from Section 4 here, since the radius of each disk is unit. Therefore, $\mathcal{D}(v)$ will denote the disk centered at $v$ of unit radius. For a vertex $v$, we use $N(v)$ instead of $N_{\text {ind }}(v)$, and for an edge $e$, we use $N(e)$ instead of $N_{\text {ind }}(e)$. As before, $D$ is the length of the longest path. Without loss of generality, we can assume that any path $P_{i}$ intersects a disk $\mathcal{D}(v)$ at most six times. If not, that is, if $P_{i} \cap \mathcal{D}(v)=\left\{v_{1}, \ldots, v_{a}\right\}$, with $a \geq 6$, we must have $d\left(v_{j}, v_{j^{\prime}}\right) \leq 1$ for some $j, j^{\prime} \leq a$, and we can construct a shorter path by "short-cutting" the segment from $v_{j}$ to $v_{j^{\prime}}$, without increasing the congestion or dilation. For a disk $S$ of radius 1 in the plane, let $C(S)$ be the number of paths $P_{i}$ that visit some vertex $v \in V$, located within $S$. Because of the this assumption, it is easy to see that $C_{\mathrm{ud}}=\max _{x \in B}\{C(\mathcal{D}(x))\}$ is within a constant factor of the congestion measure defined in Equation (3).

The main intuition for the partitioning algorithm is the following. After the first step (giving random delays) of Leighton et al. [1994], both $C_{\text {ud }}$ and $D$ become $O(\log n)$ within each time frame. This means that the smaller scheduling subproblem in any frame is localized to a $O(\log n) \times O(\log n)$ region of the plane. Thus, in addition to the 


\section{Algorithm UNITDiskEPS}

1. Run subroutine Partition $(n)$ to create smaller problems on $2 \log n \times 2 \log n$ sized grids.

2. For each of the subproblems on a $2 \log n \times 2 \log n$ sized grid, run subroutine PARTition $(2 \log n)$ to create smaller subproblems on $O(\log \log n) \times O(\log \log n)$ sized grids.

3. Solve the scheduling problem within a $O(\log \log n) \times O(\log \log n)$ sized grid by exhaustive search (details in Lemma 5.3).

4. Combine the schedules for all the subproblems together to form the whole schedule.

\section{Subroutines PARTition $(\ell)$}

INPUT A scheduling instance on a $\ell \times \ell$ region.

Output Partition this instance into smaller scheduling problems, defined on grid cells of size $2 \log \ell \times 2 \log \ell$.

1. Construct an invalid schedule $\mathcal{S}_{1}(\Pi)$ in the following manner:

(a) For each packet, choose a random delay from $\left\{1, \ldots, c\left(C_{\mathrm{ud}}+D\right)\right\}$, where $c>0$ is a specific constant, such that Lemma 5.1 is satisfied (the property in Lemma 5.1 can be checked in polynomial time; so this step involves choosing the random delays, checking the property and repeating if necessary).

(b) Allow each packet to "zip through" its path, after waiting for the random delay at the source.

2. Partition $B$ into grids $B_{2 \log \ell}^{0}, B_{2 \log \ell}^{1}, B_{2 \log \ell}^{2}$, and $B_{2 \log \ell}^{3}$.

3. Consider successive time frames of length $\log \ell$.

4. For each time frame $T$ of size $\log \ell$, assign each packet $p_{i}$ to a unique cell $Z$ in $B_{2 \log \ell}^{0}, B_{2 \log \ell}^{1}, B_{2 \log \ell}^{2}$, or $B_{2 \log \ell}^{3}$ such that the path traversed by $p_{i}$ during $T$ lies completely within $Z$; break ties arbitrarily.

5. For each time frame $T$, for each cell $Z$ in $B_{2 \log \ell}^{0}, B_{2 \log \ell}^{1}, B_{2 \log \ell}^{2}$, and $B_{2 \log \ell}^{3}$, the problem restricted to $Z$ involves scheduling the packets assigned to it during $T$, along the segments of the paths within $Z$.

Fig. 2. Algorithm for solving EPSI on unit-disk graphs.

temporal decomposition, we are able to do a spatial decomposition too. If we carry this process once more, we end up with scheduling problems on regions of size $O(\log \log n) \times$ $O(\log \log n)$, and at this point, we can solve the problem by exhaustive search in poly $(n)$ time. The algorithm is described in Figure 2 and is called Algorithm UnitDiskEPS.

Subroutine PARTITION $(\ell)$ forms a partition of the problem into smaller subproblems, each on a grid of dimension $2 \log \ell \times 2 \log \ell$.

Lemma 5.1. There exists a choice of random delays for all the packets in step 1 of subroutine PARTITION $(\ell)$ that satisfies the following property: for any time frame $T$ of length $\log \ell$, and for any lattice point $p$ in the input to the subroutine, the number of paths visiting some vertex $u \in V$ located in $S(p)$ is $O(\log \ell)$.

Proof. The input points lie in an $\ell \times \ell$ grid. By our assumption that each path visits only a constant number of vertices within $S(v)$ for any $v$, it follows that the length of the longest path, $D$, is $O(\ell)$. Let $X_{0}=C_{\mathrm{ud}}+D$. Consider any grid point $v$, and any time $t$. Then, 
$\operatorname{Pr}$ [packet $p_{i}$ passes through $\mathcal{D}(v)$ at $\left.t\right] \leq \frac{1}{c X_{0}}$ and $\mathbf{E}[\#$ packets through $\mathcal{D}(v)$ at $t] \leq 1$. By the Chernoff bound, the number of paths visiting vertices in $\mathcal{D}(v)$ during a time frame $T$ of length is $O(\log \ell)$ with probability at least $1-\ell^{-\Omega(\log \log \ell)}$. Since there are $O\left(\ell^{2}\right)$ grid points and $O(\ell)$ time frames to consider, the lemma follows by a union bound.

Lemma 5.2. In step 4 of subroutine $\operatorname{PARTITION}(\ell)$, the path traversed by any packet $p_{i}$ during a time frame $T$ (of length $\log \ell$ ) can be uniquely assigned to some cell in $B_{2 \log \ell}^{0}$, $B_{2 \log \ell}^{1}, B_{2 \log \ell}^{2}$, or $B_{2 \log \ell}^{3}$.

Proof. Let $B_{\log \ell}$ be a partition of $B$. Any packet $p_{i}$ during a time from $T$ traverses a subpath of length at most $\log \ell$. Any such path straddles at most four cells in $B_{\log \ell}$ and all such cells are adjacent to each other. Clearly, these four cells of side $\log \ell$ each are together contained in some cell in $B_{2 \log \ell}^{0}, B_{2 \log \ell}^{1}, B_{2 \log \ell}^{2}$, or $B_{2 \log \ell}^{3}$, which proves the lemma.

Lemma 5.3. A schedule of length $O(\log \log n)$ can be constructed for the scheduling problem on a grid of size $O(\log \log n) \times O(\log \log n)$.

Proof. By our assumption, each packet can only traverse $O(\log \log n)$ steps within such a grid cell, so $D=O(\log \log n)$. Also, by the guarantees of the subroutine PARTITION, $C_{\mathrm{ud}}=O(\log \log n)$ within such a grid. Therefore, the total number of packets is $O\left((\log \log n)^{3}\right)$. The maximum number of possible schedules is this number raised to the power of $C_{\text {ud }}$, which is at most a polynomial in $n$. Therefore, we can try out all schedules in polynomial time.

Corollary 5.4. A schedule for the distance-2 interference problem on unit-disk graphs of length $O(1)$ times the optimal can be found in polynomial time.

\subsection{Distributed Algorithms}

Synchronous Model. Since unit-disk graphs are a special case of disk graphs, we get the following bound as a corollary of Theorem 4.3.

Corollary 5.5. For unit-disk graphs, the EPSI problem on unit-disk graphs has a distributed algorithm with makespan $O\left(\left(C_{u d}+D\right) \cdot \log ^{2} n / \log \log n\right)$, w.h.p.

Asynchronous Model. The algorithm described in the previous section needs centralized, synchronous control, which is difficult in practice. We now describe a completely distributed, asynchronous, randomized algorithm that gives a schedule of length at most $O\left(\log ^{2} n\right)$ times the optimal w.h.p.

The basic idea is to combine contention resolution methods along with the random delays plus coloring techniques that have been used so far. Recall from the distributed approach of Section 3 that if there are $C_{\text {ud }}$ packets in the vicinity of some packet $p$ that are contending for a transmission slot at a time, all of these can be scheduled in $O\left(C_{\text {ud }} \log n\right)$ steps w.h.p. The random-delays step allows us to reduce the effective congestion at every step, and after that, one can perform coloring via the contention resolution. Note that we need to simulate some sort of synchronization to ensure that the right set of packets is contending at any time; this can easily be achieved by suitable waiting for polylogarithmic steps for each packet at each edge. The algorithm is described in Figure 3 and is referred to as Algorithm AsynchronousUnitDiskEPS.

We first discuss the following lemma, which bounds the amount of contention faced by any given packet in an interval of length $O(\log n)$. 
Algorithm AsynchronousUnitDiskEPS

1. Each packet $p_{i}$ chooses a delay $Y_{i}$ uniformly at random from $\left\{1, \ldots, \alpha_{1} X_{0}\right\}$, where $\alpha_{1}>0$ is a constant and $X_{0}=C+D$ as before.

2. Each packet waits at its source for $\left(\alpha_{2} Y_{i} \log ^{2} n\right)$ steps, where $\alpha_{2}$ is a constant.

3. Packet $p_{i}$ traverses its $\ell^{\text {th }}$ edge during the time interval $T_{i, \ell}=\left[\alpha_{2} Y_{i} \log ^{2} n+(\ell-1) \alpha_{2} \log ^{2} n+\right.$ $\left.1, \ldots, \alpha_{2} Y_{i} \log ^{2} n+\ell \alpha_{2} \log ^{2} n\right]$ as follows:

(a) Let $t \in T_{i, \ell}$ denotes the current time.

(b) If packet $p_{i}$ has already traversed its $\ell^{\text {th }}$ edge, then it keeps waiting till the end of the interval $T_{i, \ell}$.

(c) If $p_{i}$ has not yet traversed its $\ell^{\text {th }}$ edge, it chooses to traverse this edge at time $t$ with probability $\frac{1}{\alpha_{3} \log n}$, where $\alpha_{3}$ is a constant.

(d) If there is a collision during time $t, p_{i}$ retries this at the next time step.

Fig. 3. Asynchronous distributed algorithm for solving EPSI on unit-disk graphs.

Lemma 5.6. For any packet $p_{i}$ and interval $T_{i, \ell}$, there are at most $O(\log n)$ packets contending with $p_{i}$ in this interval.

Proof. Let $e=(p, q)$ denote the $\ell$ th edge on the path for packet $p_{i}$. By the definition of congestion $C_{\mathrm{ud}}$, there are at most $O\left(C_{\mathrm{ud}}\right)$ other packets that use edges in $N(e)$ (recall the definition from Section 4). Since the maximum initial random delay is $\Omega\left(C_{\mathrm{ud}}\right)$, Chernoff bounds imply that after the initial random delay, only $O(\log n)$ packets among these have $e$ as the $\ell$ th edge, w.h.p.

Lemma 5.7. Each packet $p_{i}$ moves on its $\ell$ th edge during the interval $T_{i, \ell}$ w.h.p.

Proof. Consider packet $p_{i}$ during interval $T_{i, \ell}$. The initial random delays ensure that there are at most $O(\log n)$ other packets that contend with this packet during this time interval (see Lemma 5.6). Since packets attempt to transmit at any time slot with probability $\frac{1}{\alpha_{3} \log n}$, and there are $O(\log n)$ contending packets, packet $p_{i}$ will be successful at time $t$ with probability $\frac{1}{\alpha_{3} \log n} \times\left(1-\frac{1}{\alpha_{3} \log n}\right)^{O(\log n)}$, which is $\Omega(\log n)$. Since $T_{i, \ell}$ has $\alpha_{2} \log ^{2} n$ time slots, packet $p_{i}$ transmits successfully across its $\ell^{\text {th }}$ edge w.h.p. The lemma now follows by a union bound.

Corollary 5.8. All packets are delivered within time $O\left(O P T \log ^{2} n\right)$ w.h.p., where OPT is the length of the optimal schedule.

\section{EPSI ON ARBITRARY GRAPHS}

We now handle the case of scheduling on general undirected graphs $G=(V, E)$; the maximum degree $\Delta$ of $G$ will play a key role now. Theorems 6.1 and 6.2 show nearly matching upper and lower bounds on the approximability of the problem.

We will again use the random-delays approach of Leighton et al. [1994]. As with geometric graphs, we need to define a better lower bound on the optimal makespan, since standard edge congestion is too weak: consider, for example, the complete graph $K_{n}$ with one packet to be sent on every edge; the edge congestion and dilation are each 1 , but the optimal makespan is $m=n(n-1) / 2$. Recalling that $\left\{P_{i}\right\}$ denotes the set of given routing paths, we now define

$$
C_{\mathrm{a}}=\max _{(u, v) \in E}\left(\# P_{i} \text { that involve } u+\# P_{i} \text { that involve } v\right) .
$$


Define $D$ as usual to be the dilation. Let $O P T$ denote the number of steps in the optimum schedule. It is easily seen that $O P T \geq \max \left\{C_{\mathrm{a}}, D\right\}$.

Following the notation from the first paragraph of Section 3.2, we set $q=1$ : that is, we only have one set $A_{1}$ of all SLTs. Define, for each SLT $a, I_{1}(a)$ to be the set of all other SLTs $a^{\prime}$ such that the edges used by $a$ and $a^{\prime}$ share at least one endpoint. Since each path $P_{i}$ is simple, $P_{i}$ can have at most two SLTs that involve any vertex $u$; thus, recalling Equation (4), the parameter $C^{*}$ of Section 3.2 can be taken to be $2 C_{\mathrm{a}}$. We use the initialization and invariant framework of Section 3.2, with the framescheduling algorithms to be described shortly. As in Corollary 3.3, we can take $\lambda=$ $O(\log n / \log \log n)$ so that w.h.p., for all frames $F$ and for all SLTs $a$, the number of other SLTs $a^{\prime}$ that get scheduled in $F$ and share at least one endpoint with $a$ is at most $\lambda$. Since the maximum degree of $G$ is $\Delta$, this implies that w.h.p., for all frames $F$ and for all SLTs $a$, the number of other SLTs $a^{\prime}$ that get scheduled in $F$ and interfere with $a$ (in our distance-2 sense) is at most $\beta=O(\Delta \lambda)$. Thus, we may apply the Greedy and Distributed $\left(n^{O(1)}\right)$ algorithms of Section 3.1 to obtain centralized and distributed algorithms with $L=O(\Delta \lambda)$ and $L=O(\Delta \lambda \log n)$, respectively. Since the total makespan is at most $\left(C^{*}+D\right) \cdot L=O\left(C_{\text {a }}+D\right) \cdot L$, we obtain:

THEOREM 6.1. The EPSI problem on general graphs has centralized and distributed algorithms whose respective makespans are $O\left(\left(C_{a}+D\right) \cdot \Delta \cdot \log n / \log \log n\right)$ and $O\left(\left(C_{a}+\right.\right.$ D) $\left.\cdot \Delta \cdot \log ^{2} n / \log \log n\right)$, w.h.p.

In the case where the paths are not specified, we can use the centralized algorithm of Srinivasan and Teo [2001] to obtain paths whose $C_{\mathrm{a}}+D$ is within $O(1)$ of the optimal, and schedule packets using the aforementioned algorithm. We note that the algorithm of Srinivasan and Teo [2001] is based on rounding the solution to a linear program (LP) that is designed for wired networks. However, the LP in Srinivasan and Teo [2001] can be modified to accommodate D2-edge congestion for wireless networks, in place of the plain edge congestion in wired networks.

Theorem 6.2 next demonstrates that the $O(\Delta \cdot \operatorname{polylog}(n))$-approximations of Theorem 6.1 cannot be improved to $\Delta^{1-\epsilon}$ even by centralized algorithms, unless $P=N P$.

THEOREM 6.2. Let $\epsilon$ be an arbitrary positive constant. It is not possible to approximate the optimum makespan of every instance of EPSI in polynomial time within a factor of $n^{1-\epsilon}$, unless $P=N P$. In particular, the problem is hard to approximate within $\Delta^{1-\epsilon}$.

Proof. The reduction is from Vertex Coloring. Given a graph $G(V, E)$ whose chromatic number we wish to approximate, we construct graph $G^{\prime}\left(V^{\prime}, E^{\prime}\right)$ in the following manner. For each $v \in V$, we add vertices $v, m(v)$ in $G^{\prime}$. Each edge $(u, v) \in E$ is part of $E^{\prime}$; in addition, we have edges $(v, m(v)), \forall v \in G$ in $E^{\prime}$. We have packet $p_{v}$ destined from $m(v)$ to $v$, for each $v \in V$, and the path that $p_{v}$ has to take is exactly the edge $(v, m(v))$. $\left(G^{\prime},\left\{p_{v} \mid v \in V\right\}\right)$ is our instance of PSI. We will argue that the minimum makespan of this instance of PSI is exactly the chromatic number of $G$, and this completes the proof. Two packets $p_{u}, p_{v}$ can be simultaneously delivered if and only if $(u, v) \notin E$ (and also $\left.\notin E^{\prime}\right)$, by construction of $G^{\prime}$. Therefore, the set of packets that can be simultaneously sent in a time unit corresponds to an independent set in $G$, and the time required to transmit all the packets equals the chromatic number.

Given the $n^{1-\epsilon}$-hardness of chromatic number by Zuckerman [2007], the $n^{1-\epsilon}-$ hardness of our problem follows immediately.

\section{DISTRIBUTED VERTEX COLORING}

Our packet-scheduling algorithms can be viewed as implementing a distributed coloring algorithm within each frame. This motivates the question of efficient distributed 
algorithms for various coloring problems. Luby's algorithm [Luby 1993] is a commonly used distributed coloring algorithm. One of the parameters in Luby's algorithm is the wakeup probability $w$, which needs to be at most $1 / 2$ in Luby's analysis. The empirical results of Finocchi et al. [2002] showed that Luby's algorithm improves by a constant factor on setting this value $w$ to 1 . Proving this was left as an open question in Finocchi et al. [2002], and we give a proof in this section.

We start by recapitulating Luby's vertex-coloring algorithm. Let $G$ denote the input graph; the algorithm works in the following manner. Each vertex $u \in V(G)$ is initially given a list of colors $L(u)$; initially, $|L(u)| \geq \Delta+1$, where $\Delta$ is the maximum degree in $G$. Vertices get colored using a distributed list-coloring algorithm in a synchronous roundby-round fashion (in a given round, any vertex communicates only with its neighbors). A generic round proceeds in four steps as follows:

(1) Each yet-uncolored vertex wakes up with probability $w$ and goes to sleep with the complementary probability of $1-w$.

(2) Each vertex $u$ that is awake chooses a tentative color uniformly at random from its current list $L(u)$.

(3) Each vertex $u$ that has some neighbor that chose the same tentative color as $u$ is called unsuccessful; all other (yet-uncolored) currently awake vertices are called successful.

(4) Each successful vertex $v$ is permanently given its chosen tentative color $t$, and this color $t$ is removed from $L(w)$ for all neighbors $w$ of $v$ such that $t \in L(w)$. The unsuccessful vertices proceed to the next round.

Note that once a vertex gets a permanent color, it is never considered again. Let $d_{u}$ be the degree of $u$ in the current round (in the graph induced by the yet-uncolored vertices). Let $L(u)$ be its current color list. Then it can be easily verified that $L(u) \geq d_{u}+1$. This also implies that step (2) is well defined; that is, if $u$ is yet uncolored, $|L(u)| \geq 1$. It is also easy to verify that (if and) when the algorithm terminates, $G$ has a valid vertex coloring. Let $\epsilon$ be an arbitrarily small constant and let $n$ be sufficiently large. Theorem 7.3 proves that for all graphs with $n$ vertices, if $w=1$, the aforementioned algorithm yields a valid vertex coloring within a number of rounds bounded by

$$
f(n, \epsilon) \doteq\left\lceil(1+\epsilon) \cdot \frac{\log n}{\log 1 /\left(1-(3 / 4)^{4}\right)}\right\rceil,
$$

that is, $\Theta(\log n)$, with probability at least $1-n^{-\epsilon}$. (A key idea is not just to analyze each vertex separately: for both $w=1$ and $w=1 / 2$, such an analysis can only show the same result that the probability of success for an arbitrary vertex in any round is at least $1 / 4$. Instead, Theorem 7.1 will carefully partition the vertices into subsets and show that each subset makes progress on average quite well, when $w=1$.) Complementing this, Theorem 7.4 shows that if $w=1 / 2$, then Luby's algorithm on the complete graph with $n$ vertices requires $f(n, \epsilon)+\Omega(\log n)$ rounds, with probability $1-o(1)$. Thus, setting $w=1$ is provably better in terms of worst-case performance, providing some rigorous justification for the empirical results of Finocchi et al. [2002].

A key theorem in order to prove Theorem 7.3 is the following:

THEOREM 7.1. Consider any one round of the algorithm being applied to some instance with the choice $w=1$. Let $\theta$ be the expected fraction of the yet-uncolored vertices that are successful after the round. Then, $\theta \geq(3 / 4)^{4}$.

Proof. The degree of a vertex $v$ at the start of this round is denoted $d_{v}$. Fix an arbitrary vertex $u$. Let $p_{u}$ and $p_{u}^{\prime}$ indicate the probability of vertex $u$ being successful and unsuccessful, respectively, in the current round. Suppose $u$ currently has a color 
list of size $d$; we refer to these colors as $1,2, \ldots, d$. Thus, $u$ has currently at most $d-1$ yet-uncolored neighbors $v_{1}, v_{2}, \ldots, v_{d-1}$. For $c \in\{1,2, \ldots, d\}$, let $p_{i, c}$ be the probability that $v_{i}$ picks $c$ as its tentative color. Conditioning on the tentative color choice $c$ of $u$, we get

$$
p_{u}^{\prime}=\sum_{c=1}^{d} \frac{1}{d} \cdot\left[1-\prod_{i=1}^{d-1}\left(1-p_{i, c}\right)\right]=1-\frac{1}{d} \cdot \sum_{c=1}^{d} \prod_{i=1}^{d-1}\left(1-p_{i, c}\right)
$$

Hence,

$$
p_{u}^{\prime} \leq 1-\frac{1}{d} \cdot \sum_{c=1}^{d}\left(1-\sum_{i=1}^{d-1} p_{i, c}\right)=\frac{1}{d} \sum_{c=1}^{d} \sum_{i=1}^{d_{u}} p_{i, c}=\sum_{i=1}^{d_{u}} \sum_{c=1}^{d} \frac{p_{i, c}}{d} .
$$

This leads to two useful upper bounds on $p_{u}^{\prime}$ :

$$
\begin{gathered}
p_{u}^{\prime} \leq \sum_{i=1}^{d_{u}} \sum_{c=1}^{d} \frac{p_{i, c}}{d} \leq \sum_{i=1}^{d_{u}} \max _{c=1}^{d} p_{i, c} \\
p_{u}^{\prime} \leq \frac{1}{d} \sum_{i=1}^{d_{u}} \sum_{c=1}^{d} p_{i, c} \leq \frac{1}{d} \sum_{i=1}^{d_{u}} 1=\frac{d_{u}}{d}
\end{gathered}
$$

We will now use the previous expressions to lower bound $p_{u}$. Call a vertex $v$ a highdegree vertex if and only if $d_{v} \geq 3$. Vertex $u$ will belong to at least one of the following overlapping cases:

Case 1: $d_{u} \leq 1$. In this case, Equation (8) implies that $p_{u}^{\prime} \leq 1 / 2$. Hence, $p_{u} \geq 1 / 2$.

Case 2: $d_{u}=2$. In this case, Equation (8) implies that $p_{u}^{\prime} \leq 2 / 3$. Hence, $p_{u} \geq 1 / 3$.

Case 3: $d_{u}=2$, and both neighbors of $u$ are high degree. Here, Equation (7) yields that $p_{u}^{\prime} \leq 2 \cdot \frac{1}{4}=\frac{1}{2}$. Hence, $p_{u} \geq 1 / 2$.

Case 4: $d_{u}=2$, with one neighbor of $u$ being high degree and the another neighbor having degree at least 2. In such a case, Equation (7) implies that $p_{u}^{\prime} \leq \frac{1}{4}+\frac{1}{3}=\frac{7}{12}$. Hence, $p_{u} \geq \frac{5}{12}$.

Case 5: This is a nontrivial case where vertex $u$ is a high-degree node. Recall Equation (6). We imagine an adversary who wishes to minimize $\sum_{c=1}^{d} \prod_{i=1}^{d-1}\left(1-p_{i, c}\right)$ and aim to show that this minimum cannot be "too small." What constraints does the adversary have? They are:

(C1) $\forall(i, c), p_{i, c} \geq 0$.

(C2) $\forall i, \sum_{c} p_{i, c} \leq 1$.

(C3) $\forall(i, c), p_{i, c} \leq 1 / 2$, since each $v_{i}$ has a current list of size at least 2 .

Let us study some properties of a vector of values $p_{i, c}$ that minimizes the adversary's objective function, subject to the previous constraints. (Such a minimum indeed exists, since the minimum is of a continuous function over a compact domain.) First of all, the adversary might as well replace $(\mathrm{C} 2)$ by " $\left(\mathrm{C} 2\right.$ '): $\forall i, \sum_{c} p_{i, c}=1$ ": given any feasible solution to the previous system of constraints, we can easily add some values to some of the $p_{i, c}$ to ensure (C2'), without increasing the optimal objective value OPT. We will employ the following useful claim:

CLAIM 7.2. Suppose we are given some solution with objective value OPT, where some $p_{i, c}$ is neither 0 nor $1 / 2$; then we can transform this to another solution, also with objective value OPT, which has a lesser number of $p_{i, c}$ that are neither 0 nor $1 / 2$. 
Proof. Suppose we have an optimal solution in which, say, $p_{i, c} \in(0,1 / 2)$. Then, constraints (C1), (C2'), and (C3) show that there must exist some $c^{\prime} \neq c$ such that $p_{i, c^{\prime}} \in(0,1 / 2)$. Now, consider simultaneously adding a value $\alpha$ to $p_{i, c}$ and subtracting $\alpha$ from $p_{i, c^{\prime}}$, and let $g(\alpha)$ be the net increase in the objective function from this change. It is easy to check that $g(\alpha)$ is a linear function of $\alpha$ with $g(0)=0$. Thus, one of the following two conditions should hold: (A1) $g(\alpha) \leq 0$ for all $\alpha \geq 0$, or (A2) $g(\alpha) \leq 0$ for all $\alpha \leq 0$. If (A1) holds, we will get an objective function no greater than OPT by increasing $p_{i, c}$ and decreasing $p_{i, c^{\prime}}$ (the increase and decrease being by the same value), until one of them hits 0 or $1 / 2$. Similarly, if (A2) holds, we will get an objective function no greater than $O P T$ by increasing $p_{i, c^{\prime}}$ and decreasing $p_{i, c}$ (the increase and decrease being by the same value), until one of them hits 0 or $1 / 2$.

We can repeat the argument in Claim 7.2 a finite number of times to ensure that all the $p_{i, c}$ lie in $\{0,1 / 2\}$. Now, in such a solution, let $h(c)$ be the number of $i$ for which $p_{i, c}=1 / 2$; thus, $O P T=\sum_{c=1}^{d}(1 / 2)^{h(c)}$. We can check using our earlier system of constraints that $\sum_{c} h(c)=2(d-1)$. Thus, by the convexity of the function $x \mapsto$ $(1 / 2)^{x}, O P T \geq d \cdot(1 / 2)^{2(d-1) / d} \geq d / 4$. Plugging back into Equation (6), we see that $p_{u}^{\prime} \leq 1-1 / 4=3 / 4$. Hence, $p_{u} \geq 1 / 4$.

Case 6: Vertex $u$ is a high-degree node with only high-degree neighbors. Arguments very similar to the previous case work here; let us just sketch the main differences. We still have (C1) and (C2'); instead of (C3), we have $p_{i, c} \leq 1 / 4$ since all neighbors of $u$ are high degree. Just as in Claim 7.2 , we can prove that there is an optimal solution for the adversary in which all the $p_{i, c}$ lie in $\{0,1 / 4\}$; letting $H(c)$ be the number of $i$ for which $p_{i, c}=1 / 4$, we get $O P T=\sum_{c=1}^{d}(3 / 4)^{H(c)}$. We now have $\sum_{c} H(c)=$ $4(d-1)$, and a convexity argument yields $O P T \geq d \cdot(3 / 4)^{4(d-1) / d} \geq d \cdot(3 / 4)^{4}$. Thus, $p_{u} \geq(3 / 4)^{4}$.

To complete the proof, we show next that there always exists a partition of the vertices into blocks such that the expected fraction $f$ of successful nodes in each such block is at least $(3 / 4)^{4} \sim 0.3164$. All vertices are initially free (to be put in some block). As we construct the blocks one by one, the graph remains the same, but a vertex, once selected into a block, is of course no longer free to be put into future blocks. Every time we construct a block, we also show a lower bound on $f$ (which will always be at least $(3 / 4)^{4}$ ) for that block by pointing to which case(s) earlier need to be invoked. Each new block is constructed as follows.

If there is a free high-degree node $u$ such that $u$ is connected only to other high-degree nodes (these nodes may be free or not; similar comments apply later), construct a new block $\{u\}$. Here we have $f \geq(3 / 4)^{4}$ [Case 6].

Else if there is a free pair of neighbors $u$ and $v$ such that $d_{u} \geq 3$ and $d_{v}=1$, construct a new block $\{u, v\}$. Here, $f=\left(p_{u}+p_{v}\right) / 2 \geq\left(\frac{1}{4}+\frac{1}{2}\right) / 2 \geq(3 / 4)^{4}$ [Cases 1 and 5].

Else if there is a free length-2 path $(u, v, w)$ with $d_{u} \geq 3, d_{v}=2$, and $d_{w} \geq 3$, then construct the block $\{u, v, w\}$. Here, $f \geq\left(2 \cdot \frac{1}{4}+\frac{1}{2}\right) / 3 \geq(3 / 4)^{4}$ [Cases 3 and 5].

Else if there is a free length-2 path $(u, v, w)$ with $d_{u} \geq 3, d_{v}=2$, and $d_{w}=1$, construct the block $\{u, v, w\}$. In this case, $f \geq\left(\frac{1}{4}+\frac{1}{3}+\frac{1}{2}\right) / 3 \geq(3 / 4)^{4}$ [Cases 1,2 , and 5].

Else (Comment: If none of the previous cases holds and some high-degree vertex $u$ still remains free, then it must have some free degree- 2 neighbor, whose other neighbor has degree at least 2) if there is a free pair of neighbors $u$ and $v$ such that $d_{u} \geq 3$ and $d_{v}=2$, construct a new block $\{u, v\}$. Here, $f \geq\left(\frac{1}{4}+\frac{5}{12}\right) / 2 \geq(3 / 4)^{4}$ [Cases 4 and 5 ].

Else (Comment: all remaining free vertices have degree at most 2 ) if there is a free node $u$ such that $d_{u}=2$ and with $u$ being connected only to nodes of degree at least 2 , construct a new block $\{u\}$. Herein, $f \geq 1 / 3 \geq(3 / 4)^{4}$ [Case 2]. 
Else if there is a free length-2 path $(u, v, w)$ with $d_{u} \geq 1, d_{v}=2$, and $d_{w}=1$, construct the block $\{u, v, w\}$. We have $f \geq\left(\frac{1}{2}+\frac{1}{3}+\frac{1}{2}\right) / 3 \geq(3 / 4)^{4}$ here [Cases 1 and 2].

Else (Comment: all remaining free vertices of degree 2 must have a free neighbor of degree 1 ) if there is a free pair of neighbors $u$ and $v$ such that $d_{u}=2$ and $d_{v}=1$, construct a new block $\{u, v\}$. Here, $f \geq\left(\frac{1}{2}+\frac{1}{3}\right) / 2 \geq(3 / 4)^{4}$ [Cases 1 and 2].

Else (Comment : all remaining free vertices have degree at most 1 ) if there is a free node $u$ of degree at most 1 , construct a new block $\{u\}$. In this case, $f \geq \frac{1}{2} \geq(3 / 4)^{4}$ [Case 1 ].

Thus, we have shown a lower bound of $f \geq(3 / 4)^{4}$ for each block; the linearity of expectation completes the proof of Theorem 7.1.

We are now easily led to our main result for the case $w=1$ :

THEOREM 7.3. Let $w=1, G$ be a graph with n nodes, and $\epsilon>0$ be an arbitrarily small constant. With probability at least $1-n^{-\epsilon}$, Luby's algorithm (with w set to 1 ) produces a valid vertex coloring of $G$ after $f(n, \epsilon)$ rounds, where $f$ is as in Equation (5).

Proof. Let $R_{i}$ be the number of yet-uncolored nodes after $i$ rounds. Theorem 7.1, together with induction on $i$, implies that $\mathbf{E}\left[R_{i}\right] \leq\left(1-(3 / 4)^{4}\right)^{i} \cdot n$. Letting $i=f(n, \epsilon)$, we have

$$
\mathbf{E}\left[R_{f(n, \epsilon)}\right] \leq\left(1-(3 / 4)^{4}\right)^{(1+\epsilon)} \frac{\log n}{\log 1 /\left(1-(3 / 4)^{4}\right)} \cdot n \leq n^{-\epsilon} .
$$

Since $R_{f(n, \epsilon)}$ is a nonnegative integer-valued random variable, $\operatorname{Pr}\left[R_{f(n, \epsilon)}=0\right] \geq 1-$ $\mathbf{E}\left[R_{f(n, \epsilon)}\right]$, which in turn is at least $1-1 / n^{\epsilon}$.

We next show that the worst-case performance is strictly worse when we set $w=1 / 2$, by analyzing this choice on the complete graph.

Theorem 7.4. Let $w=1 / 2$, and $K_{n}$ be the complete graph with $n$ nodes. There exist positive constants $n_{0}$ and $\epsilon$ such that for all $n \geq n_{0}$, after $f(n, \epsilon)$ rounds, the number of yet-uncolored vertices is at least $n^{k(\epsilon)}$ with probability $1-o(1)$, where $k(\epsilon)$ is a positive value independent of $n$. In particular, with $w=1 / 2$, Luby's algorithm requires $f(n, \epsilon)+\Omega(\log n)$ rounds with probability $1-o(1)$.

Proof. Since all vertices start with the same color list and symmetry and the fact that we have a complete graph imply that at the start of each round, we will have a complete graph $K_{d}$ for some $d$, with each node having the same color list of size $d$. Let $R_{i}$ denote the number of yet-uncolored vertices after $i$ rounds of the algorithm; let $\delta$ be an arbitrarily small constant. Then, the following claim holds.

Claim 7.5. There exist constants $K$ and $d_{0}=d_{0}(\delta)$ such that for any $i$ and all $d \geq d_{0}$ :

$$
\operatorname{Pr}\left[R_{i+1} \leq d(1-\delta-1 / 2 \sqrt{e}) \mid\left(R_{i}=d\right)\right] \leq 4 K /\left(\delta^{2} d\right) .
$$

Proof. Let us analyze the $(i+1)^{\text {st }}$ round, in which we work with $K_{d}$, with each node having the same color list of size $d$. We will use second-moment analysis to show that as long as $d$ is large enough, $R_{i+1}$ stays close to its mean with sufficiently large probability.

Let the vertex set be $\{1,2, \ldots, d\}$, and let $X_{u}$ be the indicator random variable for node $u$ being successful in this round. Symmetry implies that for all $u, \mathbf{E}\left[X_{u}\right]=\mathbf{E}\left[X_{1}\right]$. In addition, for all distinct nodes $u$ and $v, \mathbf{E}\left[X_{u} X_{v}\right]=\mathbf{E}\left[X_{1} X_{2}\right]$. Let $\mu$ and $\sigma$ be the mean and the standard deviation of $R_{i+1}$. It is easy to see that

$$
\operatorname{Pr}\left[X_{1}=1\right]=\frac{1}{2} \cdot(1-1 /(2 d))^{d-1},
$$

and $\mu=d \cdot \operatorname{Pr}\left[X_{1}=0\right]$. 
Let us bound the covariance of $X_{1}$ and $X_{2}$. For both $X_{1}$ and $X_{2}$ to be one,

-both nodes 1 and 2 need to be awake;

- the respective color choices $c_{1}$ and $c_{2}$ of the nodes 1 and 2 should be distinct; and

- given $c_{1}$ and $c_{2}$, none of the other $d-2$ nodes should both (1) be awake and (2) choose one of $c_{1}$ or $c_{2}$.

Thus, letting $\exp (x)$ denote $e^{x}$, we have for large $d$ that

$$
\begin{aligned}
\mathbf{E}\left[X_{1} X_{2}\right] & =(1 / 4) \cdot\left(d(d-1) / d^{2}\right) \cdot(1-(1 / 2) \cdot(2 / d))^{d-2} \\
& =(1 / 4) \cdot((d-1) / d) \cdot \exp ((d-2) \cdot \ln (1-1 / d)) \\
& =(1 / 4) \cdot((d-1) / d) \cdot \exp \left((d-2) \cdot\left(-1 / d-\Theta\left(1 / d^{2}\right)\right)\right) \\
& =(1 / 4) \cdot(\exp (-1) \pm O(1 / d)) .
\end{aligned}
$$

We next check that $\left(\mathbf{E}\left[X_{1}\right]\right)^{2}$ is close to this:

$$
\begin{aligned}
\left(\mathbf{E}\left[X_{1}\right]\right)^{2} & =(1 / 4) \cdot(1-1 /(2 d))^{2(d-1)} \\
& =(1 / 4) \cdot \exp (2(d-1) \cdot \ln (1-1 /(2 d))) \\
& =(1 / 4) \cdot \exp \left(2(d-1) \cdot\left(-1 /(2 d)-\Theta\left(1 / d^{2}\right)\right)\right) \\
& =(1 / 4) \cdot(\exp (-1) \pm O(1 / d)) .
\end{aligned}
$$

Thus, the covariance covar $\left[X_{1}, X_{2}\right]$ of $X_{1}$ and $X_{2}$ is $O(1 / d)$; the variance-covariance formula

$$
\sigma^{2}=\operatorname{var}\left[\sum_{i}\left(1-X_{i}\right)\right]=\operatorname{var}\left[\sum_{i} X_{i}\right]=\sum_{i} \operatorname{var}\left[X_{i}\right]+\sum_{(i, j): i \neq j} \operatorname{covar}\left[X_{i}, X_{j}\right]
$$

gives that $\sigma^{2} \leq d / 4+O(d)$. More precisely, there are constants $d_{1}$ and $K$ such that for all $d \geq d_{1}, \sigma^{2} \leq K \cdot d$. Also note that $(1-1 /(2 d))^{d-1}$ has a limiting value of $1 \sqrt{e}$. Hence, there exists $d_{2}=d_{2}(\delta)$ such that if $d \geq d_{2}$, then

$$
\mu \geq d \cdot(1-\delta / 2-1 /(2 \sqrt{e})) .
$$

Taking $d_{0}=\max \left\{d_{1}, d_{2}\right\}$, we thus have for all $d \geq d_{0}$ that Equation (9) holds, and that $\sigma^{2} \leq K \cdot d$.

Chebyshev's inequality implies that for all $d \geq d_{0}$,

$$
\operatorname{Pr}\left[R_{i+1} \leq d(1-\delta-1 /(2 \sqrt{e})) \mid\left(R_{i}=d\right)\right] \leq \operatorname{Pr}\left[\left|R_{i+1}-\mu\right| \geq \delta d / 2\right] \leq 4 K /\left(\delta^{2} d\right) .
$$

Thus, we have Claim 7.5.

The rest of the proof easily follows from the fact that $(3 / 4)^{4} \sim 0.3164$ is strictly greater than $1 /(2 \sqrt{e}) \sim 0.3033$. Since $(3 / 4)^{4}>1 /(2 \sqrt{e})$, we have for all sufficiently small (but positive) $\epsilon, \delta$ that

$$
n \cdot(1-\delta-1 /(2 \sqrt{e}))^{f(n, \epsilon)} \geq n^{k(\epsilon)},
$$

for some $k(\epsilon)>0$. Claim 7.5 along with induction on $i$ implies that for such sufficiently small $\epsilon$ and $\delta$,

$$
\operatorname{Pr}\left[R_{f(n, \epsilon)} \leq n(1-\delta-1 / 2 \sqrt{e})^{f(n, \epsilon)}\right] \leq \sum_{j=0}^{f(n, \epsilon)-1} 4 K /\left(\delta^{2} n(1-\delta-1 /(2 \sqrt{e}))^{j}\right)=o(1)
$$

this proves the first part of the theorem.

Since $w=1 / 2$, at most a $(1 / 2+o(1))$ fraction of the remaining vertices can get colored in any round by Luby's algorithm, w.h.p.; thus, w.h.p., Luby's algorithm requires $\Omega(\log n)$ 
additional rounds to process the remaining $n^{k(\epsilon)}$ vertices, proving the second part of the theorem.

\section{FAST DISTRIBUTED GRAPH DECOMPOSITIONS}

Recall that in the standard (synchronous) model of distributed computation, one unit of time is counted for a vertex to send and receive messages from its neighbors [Linial 1992]. A $\lambda$-decomposition of a graph $G=(V, E)$ is a partition of the vertex set into $\lambda$ subsets (called blocks). The diameter of a decomposition (or of a block of the decomposition) is the least $d$ such that any two vertices belonging to the same connected component of a block are at distance at most $d$. In the distance computation, if we allow the paths between two vertices in a connected component $S$ of a block to pass through vertices outside $S$, then the diameter is said to be weak; otherwise, the diameter is called strong. In the case of weak diameter-which is the concept of interest to us-we still are interested in it only for connected components $S$ of the blocks: we will not be concerned with the (weak) distance between vertices in different connected components. The works of Awerbuch and Peleg [1992] and Linial and Saks [1993] show that there exists a $\lambda$-decomposition of any $n$-vertex graph $G$ such that the strong diameter of each block is at most $d$, where $\lambda, d=O(\log n)$. In addition, the work of Linial and Saks [1993] provides a distributed algorithm that constructs such a decomposition with weak diameter $d$ and terminates in $O(\lambda d)$ time with high probability. We first describe this graph decomposition algorithm and then present our minor modification to it. Our modification yields the same guarantees in terms of the number of blocks and the block diameter, with improved time and message complexity. In the discussion that follows, we assume that each node $u$ has a unique $I D, I D_{u}$.

\subsection{The Linial-Saks Graph Decomposition Algorithm}

Let $d(x, y)$ denote the distance between nodes $x$ and $y$ in the given graph. The LinialSaks graph decomposition algorithm comprises several iterations. At the end of each iteration, one block gets constructed. A generic iteration proceeds as follows. Each vertex $y$ selects an integer radius $r_{y}$ at random (according to a distribution given later, which is a truncated geometric distribution). It then broadcasts $\left(I D_{y}, r_{y}\right)$ to all vertices that are within distance $r_{y}$ from it. After collecting all such messages, each vertex $y$ that is not yet part of any block selects the vertex $C(y)$ of highest ID (including itself) from among the vertices whose broadcast it received in the current iteration; $y$ joins the current block if and only if $d(y, C(y))<r_{C(y)}$-note that $d(y, C(y)) \leq r_{C(y)}$. (Vertices $y$ that are already part of some block need not do anything in this step.)

The distribution by which each vertex $x$ selects $r_{x}$ is a truncated geometric distribution, which is defined in terms of two parameters $p$ and $B$ that stay unchanged throughout the iterations:

$$
\operatorname{Pr}\left[r_{x}=j\right]= \begin{cases}p^{j}(1-p) & j \leq 0,1, \ldots B-1 . \\ p^{B} & j=B\end{cases}
$$

The following lemmas are proved by Linial and Saks [1993]. Let $G$ be any graph with at most $n$ vertices and let $S$ be the set of vertices selected at the end of one iteration of the algorithm applied to $G$.

Lemma 8.1. The weak diameter of $S$ is at most $2 B$.

Lemma 8.2. For each vertex $y$ of $G$, the probability that it belongs to $S$ is at least $p\left(1-p^{B}\right)^{n}$.

Thus, the probability of a particular vertex not being covered within $i$ iterations is at most $\left.\left(1-p\left(1-p^{B}\right)^{n}\right)\right)^{i}$; therefore, it suffices for this bound to be $o(1 / n)$ for $i=\lambda$, in 
order for the procedure to stop within $\lambda$ iterations with probability $1-o(1)$. This idea and the two previous lemmas yield the following theorem:

Theorem 8.3 [Linial AND SaKs 1993]. Let $w(n)$ be any function that tends to infinity with $n$. Letting $B=\log n+w(n) / \log (1 / p)$, with probability $1-o(1)$, the previous algorithm results in a $\lambda$-decomposition with diameter $D$ where $\lambda=(1+$ $o(1)) \log n / \log 1 /(1-p)$ and $D \leq 2 B$. In addition, the running time of the algorithm is $O(D \lambda)$.

Note that we obtain $\lambda, d=O(\log n)$ by setting $p \in(0,1)$ to be some constant, and with $w(n)=\log \log n$, say.

\subsection{Our Modification to Linial-Saks}

We now describe our small modification to the Linial-Saks algorithm. This is motivated by the graph-distance-based notion of information dissemination in distributed computing [Linial 1992], and the fact that the aforementioned algorithm of Linial and Saks [1993] really only requires each vertex to communicate with nodes of distance at most $B$ from it.

The main idea here is to parallelize the construction of all the blocks, by "simulating" the $\lambda$ iterations of the previous algorithm within a single iteration of our algorithm. At the beginning of this iteration, every vertex $y$ chooses $\lambda$ radii independently at random from the same distribution as that of Linial-Saks. Let these radii be $r_{y, 1}, r_{y, 2}, \ldots r_{y, \lambda}$, respectively. It then broadcasts $\left(I D_{y}, r_{y, 1}, r_{y, 2}, \ldots r_{y, \lambda}\right)$ to all vertices that are within distance $B$ from it. After collecting all such messages from other vertices, each vertex $y$ attempts to join one of the blocks in $\{1,2, \ldots \lambda\}$, in that order. While trying to join block $i, y$ considers all radii $r_{x, i}$, such that $r_{x, i} \geq d(x, y)$. The requirements for choosing a leader and for joining this block are the same as before. (Among all nodes $x$ that sent $y$ a message such that $r_{x, i} \geq d(x, y)$, the current leader of $y$ is the node with highest ID. Let this leader be $C_{i}(y) . y$ joins block $i$ if $d\left(y, C_{i}(y)\right)<r_{C_{i}(y), i}$; otherwise, $y$ tries to join block $i+1$.)

Recall that $r_{x}$ denotes the radius chosen by $x$ in the first round of the Linial-Saks algorithm. Let $p_{i}(y)$ be the probability of $y$ joining block $i$ in our algorithm, given that $y$ did not join blocks $1,2, \ldots, i-1$ in our algorithm. Let $p_{i}^{\prime}(y)$ be the probability of $y$ joining block $i$ in the Linial-Saks algorithm, given that $y$ did not join blocks $1,2, \ldots, i-1$ in the Linial-Saks algorithm. Our first observation is that for each $y$, all the $p_{i}(y)$ have the same value. This follows from the fact that any node $x$ chooses all its radii from the same distribution. Our next observation is that $p_{1}(y)=p_{1}^{\prime}(y)$. This follows from the fact that for the first round in both the algorithms, the joint distribution of the radii chosen by all the nodes is identical. These two observations lead to the following lemmas. $2 B$.

LEMma 8.4. The weak diameter of any block constructed in our algorithm is at most

LEMMA 8.5. For any vertex $y$ and all $i$, the probability that $y$ did not join blocks $1,2, \ldots, i$ is at most $\left(1-p\left(1-p^{B}\right)^{n}\right)^{i}$.

These two lemmas yield the following theorem:

THEOREM 8.6. Let $w(n)$ be any function that tends to infinity with $n$. Letting $B=$ $\log n+w(n) / \log (1 / p)$, our algorithm results in a $\lambda$-decomposition with diameter $D$ where $\lambda=(1+o(1)) \log n / \log 1 /(1-p)$ and $D \leq 2 B$, with probability $1-o(1)$. Moreover, the running time of our algorithm is always $O(D)$.

Corollary 8.7. Any graph $G$ can be decomposed by our algorithm into $\lambda$ blocks with weak diameter $D$ in $O(\log n)$, where $\lambda=O(\log n)$ and $D=(\log n)$. 


\section{CONCLUSION}

We have shown that generalized notions of congestion (Equations (3) and (4)) are quite useful for packet scheduling under the distance-2 interference model. Improving these bounds for more general communication requests and other objectives in addition to the makespan are interesting open problems in this interference model, as well as the SINR-based model. Extending our improved analysis of the vertex-coloring algorithm in Section 7 to more general interference models is an interesting problem.

\section{ACKNOWLEDGMENTS}

We thank Hari Balakrishnan, Venkatesan Guruswami, Subhash Khot, and Alessandro Panconesi for helpful discussions. We would also like to thank the reviewers for their detailed comments.

\section{REFERENCES}

N. Alon, F. Chung, and R. Graham. 1994. Routing permutations on graphs via matchings. SIAM Journal of Discrete Mathematics 7 (1994), 513-530.

M. Andrews. 2000. Probabilistic end-to-end delay bounds for earliest deadline first scheduling. In Proceedings of the IEEE Conference of Computer and Communications Societies (INFOCOM'00), Vol. 2. 603-612. DOI : http://dx.doi.org/10.1109/INFCOM.2000.832234

M. Andrews, A. Fernandez, M. Harchol-Balter, T. Leighton, and L. Zhang. 1997. General dynamic routing with per-packet delay guarantees of $\mathrm{O}$ (distance+1/session rate). In Proceedings of the IEEE Symposium on Foundations of Computer Science (FOCS'97). 294-302. DOI : http://dx.doi.org/10.1109/SFCS.1997.646118

F. Meyer auf der Heide, C. Schindelhauer, K. Volbert, and M. Grünewald. 2004. Congestion, dilation, and energy in radio networks. Theory of Computer Systems 37, 3 (2004), 343-370. DOI : http://dx.doi.org/10.1007/ s00224-004-1124-z

F. Meyer auf der Heide and B. Vöcking. 1995. A packet routing protocol for arbitrary networks. Lecture Notes in Computer Science 439 (1995), 291-302.

B. Awerbuch, B. Berger, L. Cowen, and D. Peleg. 1994. Low-diameter graph decomposition is in NC. Random Structures \& Algorithms 5 (1994), 441-452.

B. Awerbuch, A. V. Goldberg, M. Luby, and S. A. Plotkin. 1989. Network decomposition and locality in distributed computation. In Proceedings of the IEEE Symposium on Foundations of Computer Science. 364-369.

B. Awerbuch and D. Peleg. 1992. Routing with polynomial communication-space trade-off. SIAM Journal on Discrete Mathematics 5 (1992), 151-162.

H. Balakrishnan, C. Barrett, V. S. Anil Kumar, M. Marathe, and S. Thite. 2004. The distance-2 matching problem and its relationship to the MAC-layer capacity of ad hoc wireless networks. IEEE Journal on Selected Areas in Communications (JSAC) 22, 6 (2004), 1069-1079.

V. Bonifaci, P. Korteweg, A. Marchetti-Spaccamela, and L. Stougie. 2011. Minimizing flow time in the wireless gathering problem. ACM Transactions on Algorithms 7, 3, Article 33 (July 2011), 20 pages. DOI : http://dx.doi.org/10.1145/1978782.1978788

D. Chafekar, V. S. Anil Kumar, M. V. Marathe, S. Parthasarathy, and A. Srinivasan. 2007. Cross-layer latency minimization in wireless networks with SINR constraints. In Proceedings of the ACM International Symposium on Mobile Ad Hoc Networking and Computing (MobiHoc'07). 110-119.

H. Chernoff. 1952. A measure of asymptotic efficiency for tests of a hypothesis based on the sum of observations. Annals of Mathematical Statistics 23 (1952), 493-509.

J. Dams, M. Hoefer, and T. Kesselheim. 2012. Scheduling in wireless networks with rayleigh-fading interference. In Proceedings of the ACM Symposium on Parallelism in Algorithms and Architectures (SPAA'12).

M. Dinitz. 2010. Distributed algorithms for approximating wireless network capacity. In Proceedings of the 29th Conference of the IEEE Communications Society (INFOCOM'10). 1397-1405.

D. Dubhashi, A. Mei, A. Panconesi, J. Radhakrishnan, and A. Srinivasan. 2005. Fast distributed algorithms for (weakly) connected dominating sets and linear-size skeletons. Journal of Computer and System Sciences 71, 4 (2005), 467-479. DOI : http://dx.doi.org/10.1016/j.jcss.2005.04.002

A. Fanghänel, T. Kesselheim, and B Vöcking. 2009. Improved algorithms for latency minimization in wireless networks. In Proceedings of the International Colloquium on Automata, Languages and Programming (ICALP'09). 447-458. 
I. Finocchi, A. Panconesi, and R. Silvestri. 2002. Experimental analysis of simple, distributed vertex coloring algorithms. In Proceedings of the 13th Annual ACM-SIAM Symposium on Discrete Algorithms. Society for Industrial and Applied Mathematics, 606-615.

D. A. Grable and A. Panconesi. 2000. Fast distributed algorithms for brooks-vizing colorings. Journal of Algorithms 37 (2000), 85-120.

M. M. Halldórsson and P. Mitra. 2011. Nearly optimal bounds for distributed wireless scheduling in the SINR model. In Proceedings of the International Colloquium on Automata, Languages and Programming (ICALP'11).

M. M. Halldórsson and T. Tonoyan. 2015. How well can graphs represent wireless interference? In Proceedings of the ACM Symposium on Theory of Computing (STOC'15).

M. M. Halldórsson and R. Wattenhofer. 2009. Wireless communication is in APX. In International Colloquium on Automata, Languages and Programming (ICALP'09). 525-536.

W. Hoeffding. 1963. Probability inequalities for sums of bounded random variables. American Statistical Association Journal 58 (1963), 13-30.

T. Kesselheim. 2012. Approximation algorithms for wireless link scheduling with flexible data rates. In Proc. European Symposium on Algorithms (ESA'12).

S. O. Krumke, M. V. Marathe, and S. S. Ravi. 2001. Models and approximation algorithms for channel assignment in radio networks. Wireless Networks 7, 6 (2001), 575-584. DOI:http://dx.doi.org/10.1023/ A:1012311216333

V. S. Anil Kumar, M. Marathe, and S. Parthasarathy. 2008. Cross-layer capacity estimation and throughput maximization in wireless networks. Handbook on Algorithms for Next Generation Networks (2008).

V. S. Anil Kumar, M. V. Marathe, S. Parthasarathy, and A. Srinivasan. 2004. End-to-end packet scheduling in Ad hoc networks. In Proc. ACM SIAM Symposium on Discrete Algorithms (SODA'04).

V. S. Anil Kumar, M. V. Marathe, S. Parthasarathy, and A. Srinivasan. 2005. Algorithmic aspects of capacity in wireless networks. In Proc. of the ACM International Conference on Measurement and Modeling of Computer Systems (SIGMETRICS'05). ACM, New York, NY, 133-144. DOI : http://dx.doi.org/10.1145/ 1064212.1064228

F. T. Leighton, B. M. Maggs, and A. W. Richa. 1999. Fast algorithms for finding O(Congestion + Dilation) packet routing schedules. Combinatorica 19, 2 (1999), 1-27.

T. Leighton, B. Maggs, and S. Rao. 1994. Packet routing and job shop scheduling in O(Congestion+Dilation) steps. Combinatorica 14, 2 (1994), 167-180.

N. Linial. 1992. Locality in distributed graph algorithms. SIAM Journal on Computing 21 (1992), $193-201$.

N. Linial and M. Saks. 1993. Low diameter graph decompositions. Combinatorica 13 (1993), 441-454.

Z. Lotker and D. Peleg. 2010. Structure and algorithms in the SINR wireless model. ACM SIGACT News (2010).

M. Luby. 1993. Removing randomness in parallel computation without a processor penalty. Journal of Computer and System Sciences 47, 2 (1993), 250-286.

R. Ostrovsky and Y. Rabani. 1997. Universal $\mathrm{O}\left(\right.$ Congestion + Dilation $\left.+\log ^{1+\epsilon}(N)\right)$ local control packet switching algorithms. In Proceedings of the 29th Annual ACM Symposium on Theory of Computing. ACM, 644-653. DOI : http://dx.doi.org/10.1145/258533.258659

A. Panconesi and A. Srinivasan. 1996. On the complexity of distributed network decomposition. Journal of Algorithms 20, 2 (1996), 356-374. DOI : http://dx.doi.org/10.1006/jagm.1996.0017

G. Pei and A. K. S. Vullikanti. 2012. Brief announcement: Distributed algorithms for maximum link scheduling under the physical interference model. In Proceedings of the International Symposium on Distributed Computing (DISC'12).

L. Peterson and B. Davie. 2000. Computer Networks: A Systems Approach. Morgan Kaufman Publishers.

S. Ramanathan. 1993. Scheduling Algorithms for Multi-Hop Radio Networks. (1993). Ph.D. thesis, Department of Computer Science, University of Delaware.

S. Ramanathan. 1999. A unified framework and algorithm for channel assignment in wireless networks. Wireless Networks 5, 2 (1999), 81-94. DOI: http://dx.doi.org/10.1023/A:1019126406181

S. Ramanathan and E. L. Lloyd. 1993. Scheduling algorithms for multihop radio networks. IEEE/ACM Transactions on Networking 1, 2 (1993), 166-177. DOI : http://dx.doi.org/10.1145/153377.153379

S. Ruhrup, C. Schindelhauer, K. Volbert, and M. Grunewald. 2003. Performance of distributed algorithms for topology control in wireless networks. In Proceedings of the IEEE International Parallel and Distributed Processing Symposium (IPDPS'03).

C. Scheideler. 1998. Universal routing strategies for interconnection networks. Lecture Notes in Computer Science 1390 (1998). 
A. Srinivasan and C.-P. Teo. 2001. A constant-factor approximation algorithm for packet routing and balancing local vs. global criteria. SIAM Journal on Computing 30, 6 (2001), 2051-2068. DOI :http://dx.doi.org/ 10.1137/S0097539798335596

P.-J. Wan, X. Jia, and F. Yao. 2009. Maximum independent set of links under physical interference model. In Proceedings of Wireless Algorithms, Systems, and Applications (WASA). 169-178.

D. Zuckerman. 2007. Linear degree extractors and the inapproximability of max clique and chromatic number. Theory of Computing 3, 1 (2007), 103-128. DOI : http://dx.doi.org/10.4086/toc.2007.v003a006

Received July 2012; revised July 2015; accepted August 2015 